\title{
Charting the Lower Superior Temporal Region, a New Motion-Sensitive Region in Monkey Superior Temporal Sulcus
}

\author{
Koen Nelissen, ${ }^{1}$ Wim Vanduffel,,${ }^{1,2}$ and Guy A. Orban ${ }^{1}$ \\ ${ }^{1}$ Laboratorium voor Neurofysiologie en Psychofysiologie, K.U. Leuven Medical School, Campus Gasthuisberg, 3000 Leuven, Belgium, and ${ }^{2}$ Massachusetts \\ General Hospital, Massachusetts Institute of Technology, Harvard Medical School, Athinoula A. Martinos Center for Biomedical Imaging, Charlestown, \\ Massachusetts 02129
}

\begin{abstract}
Although the role of the middle temporal (MT/V5) area and its medial superior temporal (MST) satellites in motion processing has been well explored, relatively little is known about motion regions located more rostrally in the superior temporal sulcus (STS), such as the fundus of the superior temporal (FST) area, the superior temporal polysensory (STP) region, or beyond. To fill this void, we used contrast-enhanced functional magnetic resonance imaging in awake macaques and a five-step testing procedure that allowed us to identify six motion-sensitive regions within the STS. Direction adaptation tests confirmed the motion sensitivity of these six regions. Five of them [MT/V5, its three satellites, and the middle part of the STP (STPm) region in the upper bank of the STS] have been documented by previous single-cell studies. A sixth, previously unknown motion-responsive region, which we termed the lower superior temporal (LST) region, was observed on the lower bank and fundus of the STS, 6-8 mm anterior to the FST area. In contrast to the MST areas, the LST region responds to slow as well as fast speeds and is responsive to static and moving images of objects, to patterns defined by opponent motion, and to actions. These results, obtained in both group and single-subject analyses, suggest that motion information in the STS might follow a second path, in addition to the MT/V5-MST path. This ventral path including the LST region, FST area, and STPm region is likely involved in the visual analysis of actions and biological motion.
\end{abstract}

Key words: vision; cerebral cortex; functional imaging; primates; action; cortical area

\section{Introduction}

A number of motion-sensitive regions have been described in the macaque superior temporal sulcus (STS). The middle temporal (MT/V5) area is identified by its high proportion of directionselective cells, direct input from V1, retinotopic representation of the entire contralateral hemifield, and heavy myelination (Dubner and Zeki, 1971; Zeki, 1974; Ungerleider and Desimone, 1986a; Maunsell and Van Essen, 1987). Several satellites of MT/V5 have been described including the dorsal medial superior temporal (MSTd), ventral MST (MSTv), and fundus of the superior temporal (FST) areas (Desimone and Ungerleider, 1986; Komatsu and Wurtz, 1988; Tanaka et al., 1993). The extent of the FST area is unclear, however, as is the exact partitioning of the

Received July 20, 2005; revised April 18, 2006; accepted April 20, 2006.

This work was supported by grants from the Queen Elisabeth Medical Foundation (GSKE), the National Research Foundation (FWO G 0151.04), the Flemish Regional Ministry of Education (GOA 2005/18), InterUniversity Attraction pole P5/04, Mirror (European Union Life Science), the Human Frontier Science Program, and the MIND Institute. We thank W. Depuydt, M. De Paep, A. Coeman, C. Fransen, P. Kayenbergh, G. Meulemans, Y. Celis, and G. Vanparrys (deceased, May, 2005) for technical support and D. Fize, W. van Beerendonk, H. Sawamura, and J.-B. Durand for help with the scanning and analysis. We also thank $\mathrm{S}$. Raiguel for comments on this manuscript and $\mathrm{G}$. Rizzolatti for providing the action videos. The Laboratoire Guerbet (Roissy, France) provided the contrast agent (Sinerem).

Correspondence should be addressed to Dr. Guy A. Orban, Laboratorium voor Neurofysiologie en Psychofysiologie, K.U. Leuven Medical School, Campus Gasthuisberg, Herestraat 49, 3000 Leuven, Belgium. E-mail: guy.orban@med.kuleuven.be.

DOI:10.1523/JNEUROSCI.0824-06.2006

Copyright $\odot 2006$ Society for Neuroscience $\quad$ 0270-6474/06/265929-19\$15.00/0
MST area (for review, see Van Essen, 2004). In addition, motion sensitivity has been reported in the superior temporal polysensory (STP) region, located on the upper bank of the STS in front of the MSTd area (Bruce et al., 1981; Perrett et al., 1985; Hikosaka et al., 1988). It has been difficult to pin down a precise location for motionsensitive neurons in the STP region, and they are generally assumed to be located in the rostral part of the STP region (for review, see Cusick, 1997). In addition, visual responses to actions have been documented in both the upper and the lower bank of the STS (Perrett et al., 1989). Thus, beyond MT/V5, the exact partitioning of motion-sensitive STS regions remains elusive.

The reasons for the discrepancies between the various schemes for partitioning the STS are twofold: the four criteria defining cortical areas (cytoarchitecture and myeloarchitecture, connectivity, retinotopic organization, and functional properties) apply only incompletely to the STS regions, and the schemes are extrapolations from many studies using different techniques and different subjects. Functional magnetic resonance imaging (fMRI) in the macaque (Logothetis et al., 1999) allows extensive functional testing in the same individual. Hence, fMRI provides an opportunity to define visual cortical regions of the macaque in a systematic and coherent manner. In humans, attempts have been made to parcel the human MT/V5 complex (Dukelow et al., 2001; Huk et al., 2002), but these have been restricted to identifying the homolog of the MST area. 
To chart the motion-sensitive STS regions, we used contrast agent-enhanced fMRI in awake monkeys (Vanduffel et al., 2001). First, we outline the motion-responsive cortex in general and chart specific areas within this motion-sensitive cortex, using subtractions based on speed, optic flow selectivity, and receptive field (RF) sizes as documented by single-cell studies of the MT/V5 and MST areas (Maunsell and Van Essen, 1983; Desimone and Ungerleider, 1986; Mikami et al., 1986; Saito et al., 1986; Duffy and Wurtz, 1991; Lagae et al., 1994; Orban et al., 1995; Raiguel et al., 1997). Next, we characterize the charted regions by their fMRI adaptation for direction of motion, suggesting the presence of direction-selective neurons. Finally, we further investigate the function of these regions using the MR responses to a battery of motion stimuli, including simple translation and complex stimuli such as kinetic boundaries (Regan, 1989), three-dimensional structure-from-motion displays (Todd, 1984), or action videos (Grafton et al., 1996).

\section{Materials and Methods}

Subjects

Five male rhesus monkeys (M1, M3, M4, M5, and M6), 4-6 kg in weight, participated in the present experiments. The surgical details and procedures of the training of the monkeys were described previously (Vanduffel et al., 2001; Fize et al., 2003; Nelissen et al., 2005). The surgical and other procedures conformed to national, European, and National Institutes of Health guidelines for the care and use of laboratory animals.

During scanning, the monkey subjects sat in the sphinx position in a plastic monkey chair directly facing the screen, onto which stimuli were projected. Eye position was monitored (at $50 \mathrm{~Hz}$ ) through the pupil position and the corneal reflection (Iscan, Burlington, MA). The monkey was rewarded for maintaining fixation within $1^{\circ}$ of a small red fixation spot $\left(0.3 \times 0.3^{\circ}\right)$ while stimuli were projected in the background. The interval between rewards was systematically decreased (from 2500 to 500 $\mathrm{ms})$ as the monkey maintained his fixation within the window. During scanning, this window was elongated to $3^{\circ}$ in the vertical direction to accommodate occasional artifacts on the vertical eye movement recording. A radial receive-only surface coil $(10 \mathrm{~cm}$ diameter $)$ was positioned immediately over the head.

Immediately before the scanning, a contrast agent, monocrystalline iron oxide nanoparticle (MION; $5-11 \mathrm{mg} / \mathrm{kg}$ ) diluted in sodium citrate buffer, $\mathrm{pH}$ 8.0, was injected intravenously into the femoral or external saphenous vein. In later experiments, the same contrast agent, produced under a different name (Sinerem; Laboratoire Guerbet, Roissy, France), was used. The use of a contrast agent improved both the contrast-tonoise ratio (by approximately fivefold) and the spatial specificity of the signal changes compared with blood oxygenation level-dependent (BOLD) measurements (Vanduffel et al., 2001; Leite et al., 2002). Indeed, it has been repeatedly reported that MION MR signals, which depend on blood volume, reach a maximum in the middle of the cortex, in layer 4, whereas BOLD signals are maximum at the cortical surface arising from pial vessels (Mandeville and Marota, 1999; Harel et al., 2002; Zhao et al., 2006). Recent evidence indicates that blood volume changes occur in the microvessels, joining the small arteries penetrating the cortex and the cortical capillaries (Zhao et al., 2006). Furthermore, blood volume is regulated at the submillimeter level along both the radial direction of the cortex and the cortical surface (Zhao et al., 2005, 2006).

\section{Visual stimuli}

Visual stimuli were projected from a Barco 6300 liquid crystal display projector $(1280 \times 1024$ pixels; $60 \mathrm{~Hz}$ refresh rate) using customized optics (Buhl Optical, Rochester, NY) onto a screen that was positioned 54 $\mathrm{cm}$ in front of the monkey's eyes. Luminance of the stimuli was $2.0 \mathrm{~cd} / \mathrm{m}^{2}$ for white parts and $0.1 \mathrm{~cd} / \mathrm{m}^{2}$ for black parts. Two tests were used in three monkeys (M1, M3, and M4) to localize the motion-responsive cortex: the optic flow (2100 volumes in a single session in each monkey) and speed ( 2250 volumes in one or two sessions in each monkey) tests. In the optic flow test, random dot patterns $\left(25 \%\right.$ white $0.3^{\circ}$ dots on a black background) were presented in a circular aperture $22^{\circ}$ in diameter. These patterns were either expanding/contracting, rotating (clockwise and counterclockwise), translating (eight different directions), or stationary (Lagae et al., 1994). To maintain dot density, a fraction of the dots $(<1 \%$ per frame) were replotted, including in the static condition, which is therefore referred to as "static plus" condition. In the speed test, random textured patterns (RTP; $50 \%$ white $0.075^{\circ}$ dots, $50 \%$ black $0.075^{\circ}$ dots) were presented in a circular aperture $\left(14^{\circ}\right.$ diameter $)$ either stationary or translating in eight different directions along the four main axes (Vanduffel et al., 2001) at five different speeds $(1,2,4,8$, and 16\%). For the purpose of parceling the motion-responsive cortex based on functional properties, two additional tests were used. In the opponent motion grating test (M1 and M3, each 3200 volumes in two sessions), kinetic gratings were presented consisting of RTPs $\left(14^{\circ}\right.$ circular aperture $)$ in which alternating bands moved in opposite directions (Orban et al., 1995; Van Oostende et al., 1997). The motion direction in these stimuli was parallel to the boundaries that remained stationary. The width of the stripes was either $1 / 8,1 / 4,1 / 2,1,2$, or $4^{\circ}$. Two additional stimuli were used in this test, transparent motion (a special case of the kinetic grating stimuli in which the width of the stripes is reduced to 1 pixel) and a stationary RTP. In the moving shapes test (M3 and M5, each 3600 volumes in one or two sessions), we used the grayscale images from the study by Kourtzi and Kanwisher (2000) (see also Denys et al., 2004), but they were reduced in size to $7^{\circ}$ diameter. These images were presented on a $15 \times 15^{\circ}$ white square background and were either translating along the four main axes (eight directions) or static in the center. As a control, the scrambled counterparts were presented, either translating or stationary. In the fixation-only condition, only the white background was presented.

To describe the basic properties of the motion-sensitive regions, we investigated both the retinotopic organization and the direction-selective adaptation (Huk et al., 2001). In all five monkeys, a standard retinotopic exploration was performed with the tests described by Fize et al. (2003). The results in M6 were identical to those reported by Fize et al. (2003) for the four other monkeys. In M6, we used the eye position signal to split the data into two parts depending on the quality of fixation, but this yielded very little difference in the retinotopic maps obtained. The center and peripheral field projections were explored in the eccentricity test (M1 and M5, 4200 volumes each in one or two sessions) and used two RTPs: a central circular aperture of $2^{\circ}$ diameter and an annulus with inner and outer diameters of 7 and $14^{\circ}$, respectively. These stimuli were presented either stationary or moving at 1 or $16^{\circ}$ s. The direction adaptation test (M5 and M6, 1450 volumes each in two sessions) was adapted from Huk et al. (2001). Basically, after a 36 s block of adaptation, we alternated five times $24 \mathrm{~s}$ blocks in which motion was presented either in directions differing by 45,90 or $135^{\circ}$ from the adapted direction (mixed blocks) or in the adapted direction (adapted blocks). Before the initial adaptation block and after the last test block, a 36 s fixation baseline block was included. In the mixed and adapted motion blocks, 24 motion periods of $0.750 \mathrm{~s}$ were presented interspersed with an equal number of $0.250 \mathrm{~s}$ fixation periods. Random dot patterns (same patterns as in the optic flow test) moved in opposite direction in the two visual fields, either within a single central circular aperture $\left(7^{\circ}\right.$ diameter) or in two $10^{\circ}$ apertures centered at $5^{\circ}$ eccentricity on the horizontal meridian in each visual field. Although it could be argued that the mixed and adapted blocks also differ in residual orientation (as a result of the large dot size), these effects must be small because of the broad tuning of MT/V5 and MSTd neurons for speed (Lagae et al., 1993; Orban et al., 1995).

For further functional characterization, six additional tests were used. The aim of these tests was to explore additional parameters of simple translating RTPs and higher-order motion stimuli. Just as optic flow stimuli have been helpful for investigating the MST areas, we reasoned that other types of stimuli such as opponent motion or kinetic stimuli (Sary et al., 1993; Unno et al., 2002), three-dimensional structure-frommotion stimuli (Vanduffel et al., 2002), and action stimuli (Perrett et al., 1989) would reveal the function of the other motion-sensitive regions beyond MT/V5. In the size test (M1 and M5, 3240 volumes each in one session), RTPs of four different sizes (circular apertures $3,7,14$, and $28^{\circ}$ in diameter) were presented. These RTPs were either stationary or moving (speed $4 \%$ ) along the four main axes (eight directions), as in the speed test. In the shape-from-motion test (M1, M3, and M5, 3600 vol- 
umes each in two to four sessions), RTPs were used to generate kinetically defined shapes ( $4^{\circ}$ diameter). The eight different shapes are those used initially by Sary et al. (1993) in their inferotemporal (IT) study. Transparent motion stimuli and RTPs moving uniformly in a single direction per presentation (uniform motion), as in the speed test, were used as additional controls. In the three-dimensional structure-from-motion test (M1, M3, and M5, 2880 volumes each in four sessions), we presented monocular stimuli $\left(10^{\circ}\right.$ diameter $)$ that consisted of nine interconnected lines of random length $\left(4.5^{\circ}\right.$ average $)$ and orientation. As in the study by Vanduffel et al. (2002), they were presented either stationary, translating along the horizontal axis (in fixation plane), or rotating in depth along the vertical axis. It should be noted that in the study by Vanduffel et al. (2002), the lines made right angles with one another, rather than the arbitrary angles as used here.

Finally, three action tests were also administered: the action test, the goal-directed action test, and the action-moving object test as described by Nelissen et al. (2005). In the action test (M1, M3, and M5, 3240 volumes each in 14 sessions in total), identical to the action test of experiment 2 of Nelissen et al. (2005), three conditions were compared: action (videos showing male or female hand actions, precision, or whole-hand grasp), static (single frame from the middle of the video shown for $36 \mathrm{~s}$ ), and scrambled (dynamic stimulus produced by phase scrambling of each frame of the video sequences). In the goal-directed action test (M3 and M5, 4200 volumes each in two sessions), identical to the second test in experiment 3 of Nelissen et al. (2005), we presented videos showing hands grasping objects (goal-directed actions) and their static control (a frame from the middle of the video) and hands making a grasping movement without an object (mimicking action) and their static control in a $2 \times 2$ factorial design. In the action-moving object test (M3 and M5, 4200 volumes each in two and three sessions, respectively), identical to the test in experiment 2 of Nelissen et al. (2005), we compared action hand, static hand, moving object, and static object in a $2 \times 2$ factorial design.

\section{Scanning}

Monkeys were scanned in a horizontal bore, 1.5 T MR scanner (Sonata; Siemens, Erlangen, Germany) equipped with echoplanar imaging. Each functional time series consisted of gradient-echo echoplanar wholebrain images: repetition time (TR), $2.4 \mathrm{~s}$; echo time (TE), $27 \mathrm{~ms} ; 64 \times 64$ matrix; $2 \times 2 \times 2 \mathrm{~mm}$ voxels ( 32 sagittal slices). A block design was used in all experiments ( 24 or $36 \mathrm{~s}$ blocks). The number of conditions per time series varied between tests from four to nine. Within the same time series, the presentation order of the conditions was repeated two or three times. Between time series, the order of the conditions was randomized.

For each subject, a high-resolution anatomical image (magnetization prepared rapid acquisition gradient echo) was acquired (TR, $1950 \mathrm{~ms}$; TE, $3.9 \mathrm{~ms}$; inversion time, $800 \mathrm{~ms} ; 240 \times 256$ matrix; $1 \times 1 \times 1 \mathrm{~mm}$ voxels; 160 sagittal slices).

A total of 43,710 volumes were scanned during 22 sessions in M1; 21,095 volumes were scanned in 11 sessions in M3; 13,180 volumes were scanned in 6 sessions in M4; 66,235 volumes were scanned in 37 sessions in M5; and 4205 volumes were scanned in 5 sessions in M6. Monkeys M1 and M5 were the subjects participating in most of the tests.

\section{Data analysis}

Volume-based analyses. Data were analyzed using statistical parametric mapping (SPM 99), Statistica (Statsoft, Tulsa, OK), and Match software. Only scans in which the monkeys held their fixation within the $2 \times 3^{\circ}$ window for $>80 \%$ of the time were analyzed. In these analyses, realignment parameters, as well as eye movement traces, were included as covariates of no interest to remove eye movement and brain-motion artifacts. Furthermore, statistical testing compared the number of saccades in the different conditions of a test. The monkey functional volumes were realigned and nonrigidly coregistered with their anatomical volumes using a customized volume-based registration algorithm, Match (Chefd'Hotel et al., 2002). The algorithm computes a dense deformation field by composition of small displacements minimizing a local correlation criterion. Regularization of the deformation field is obtained by low-pass filtering. This registration benefited from the high contrast-tonoise ratio in the functional volumes resulting from the use of the con- trast agent. The monkey functional volumes were then subsampled to 1 $\mathrm{mm}^{3}$ and smoothed with an isotropic Gaussian kernel (full width at half height, $1.5 \mathrm{~mm}$ ). The fMRI data of each daily session of the individual monkeys were registered to a single standard brain anatomy (M3) using the Match software (Chef d'Hotel et al., 2002). The quality of the realignment can be appreciated from supplemental Fig S6 in the study by Nelissen et al. (2005)

All statistical analyses, which include averaging across sessions, were performed either on group data (fixed effects) of two to four monkeys or on the data from individual subjects. The variability between monkeys was small enough and the elastic registration to the template was powerful enough to allow definition of areas on the more representative group data. Therefore, it is primarily the group data that are reported here. The threshold for significance was set to $p<0.05$ corrected for multiple comparisons in all tests, unless mentioned otherwise.

Region of interest-based analyses. Based on the differential activation in the four basic tests, six regions of interest (ROIs) were defined in the left and right hemisphere of M3. The ROIs included the left and right MT/V5 (30 and 26 voxels, respectively), left and right MSTv (30 and 21 voxels), left and right MSTd (30 and 16 voxels), left and right FST (23 and 29 voxels), left and right lower superior temporal (LST) region (15 and 20 voxels), and left and right STPm (middle part of the STP region; 17 and 18 voxels). Activity profiles plotting the percentage of MR signal changes for the different conditions of a test were calculated from the group data in the ROI as a whole or within each of the voxels of the ROIs. In both cases, the data of the first two volumes of a block were omitted to take into account the hemodynamic delay.

We performed two statistical tests on the data of each fMRI test. The first type of statistic, the temporal statistic, compared the overall activity in the ROI, averaged over the voxels, as a function of time and tested how well it followed the experimental paradigm. Significance was assessed with either a one-way ANOVA or a factorial ANOVA (Statistica; Statsoft), followed by a post hoc test correcting for the number of conditions in the test. Next, we compared the activity averaged over time in the different conditions across the unsmoothed voxels of the ROI in a spatial analysis. The same ANOVA tests were used as those in the temporal statistics. This spatial statistic probes for coherency across the ROI and was more stringent than the temporal statistic in all tests. Therefore, this statistic is reported in Tables $2-4$. In these tables, we retain as significant those subtractions reaching $p<0.05$ both in the group and in at least two-thirds of the single subjects.

\section{Flattening and sampling of the STS}

To avoid the deformations induced by flattening the entire hemisphere (Van Essen, 2005), most of the data will be presented on a reconstructed flattened representation of the isolated STS of monkey M3. Using this approach, we minimize the local deformation of the STS while retaining optimal registration between functional and anatomical images of the STS. The full extent of the STS is indicated by the dashed white line in Figure 1, $A$ and $B$ (1, anteroventral end of the STS; 2 , posterodorsal end of the STS). The gray zone in Figure $1 B$ indicates the part of the left STS sampled in this study (from $7 \mathrm{~mm}$ posterior to $16 \mathrm{~mm}$ anterior relative to the interaural plane). The data represented on the reconstructed flat map were sampled directly onto the coronal sections of the MRI anatomy. The sampling is illustrated on the coronal sections at different anteroposterior levels $(+12,+4,-2$, and $-6 \mathrm{~mm}$ relative to the interaural plane) (Fig. 1C). The gray matter was sampled at mid-depth in steps of $1 \mathrm{~mm}$ from the lip of the lower bank (indicated by the arrows) through the fundus, to the lip of the upper bank (indicated by the arrowheads). The sampling points acquired between the two green, blue, yellow, and gray arrows and arrowheads correspond to the positions indicated by the same symbols in the flat map shown in Figure $1 \mathrm{~B}$. In the coronal sections, the distance between the centers of two neighboring voxels is either $1 \mathrm{~mm}$ (voxels lying next to each other horizontally or vertically) or $1.41 \mathrm{~mm}$ (neighboring voxels lying diagonally). Hence, at a given anteroposterior level (coronal section) on the flat maps, neighboring voxels are presented without or with a small gap. For clarity of illustration, the different anteroposterior levels (from -7 to $+16 \mathrm{~mm}$ ) were spaced at a fixed distance. To stack the voxels from successive coronal sections, we aligned 
the fundus in the different sections. This minimized distortions because the fundus was located at the same laterality ( $18 \mathrm{~mm}$ from midline) in most sections. Only in the two most rostral and the four most caudal sections did the STS move medially. To correct this, we used increasingly more medial points of the fundus for aligning the last four sections $(-4$ to -7$)$, reaching point "b" (Fig. 1C) in the last section. This almost perfectly compensated the change in laterality of the fundus.

Notice that part of the caudal STS (Fig. $1 B$, dark gray zone between $\mathrm{b}$ and $\mathrm{c}$ at levels -5 to $-7 \mathrm{~mm}$ relative to the interaural plane) corresponds to a small variable sulcus extending from the posterior parietal cortex, which was present in three of the five monkeys studied (including the template M3) (supplemental Fig. S1, available at www.jneurosci.org as supplemental material). Examination of the anatomical MRI data of 13 other monkeys showed that this sulcus was present in 8 of 13 subjects ( 16 of 26 hemispheres). As will be shown below, in most cases, this part of the cortex displayed little significant MR signal change for moving compared with static stimuli.

\section{Results}

Behavioral results

The five monkeys participating in the study fixated well. On average, over the different tests, the different monkeys made 11 saccades/min (range, 6-22 saccades/ min) (Table 1). More importantly, this number did not differ significantly between conditions in most ( 21 of 26$)$ tests (Table 1). In the few tests in which a monkey made significantly different numbers of saccades between conditions, this was generally attributable to an increased number of saccades in the control fixation condition, with no difference between the experimental conditions. The single exception was M5 in the speed test, in which the monkey made more saccades during the $16 \%$ and static conditions than during the other conditions. Thus, eye movements are very unlikely to account for our fMRI results.

\section{Localizing the motion-responsive cortex in the STS}

A first series of analyses was aimed at identifying the portions of the STS responding to visual motion in general. We defined five contrasts derived from the optic flow and speed tests. We contrasted slow $(1 \% \mathrm{~s})$ and fast $(16 \%)$ moving RTPs, as well as expanding/contracting, rotating, and translating random dot patterns with their respective stationary controls. Notice that in the optic flow test but not the speed test, the static and motion stimuli included some flicker (static plus condition). Figure 2 shows all voxels significant ( $p<0.05$, corrected) for at least one of these five contrasts for the left and right hemispheres. To make comparisons with previously published results (Vanduffel et al., 2001), we located the SPM local maxima (Fig. 2, white circles) for these five motion contrasts within the STS. Based on the study by Vanduffel et al. (2001), three of these local maxima could be identified as corresponding to the central MT/V5, MSTv, and

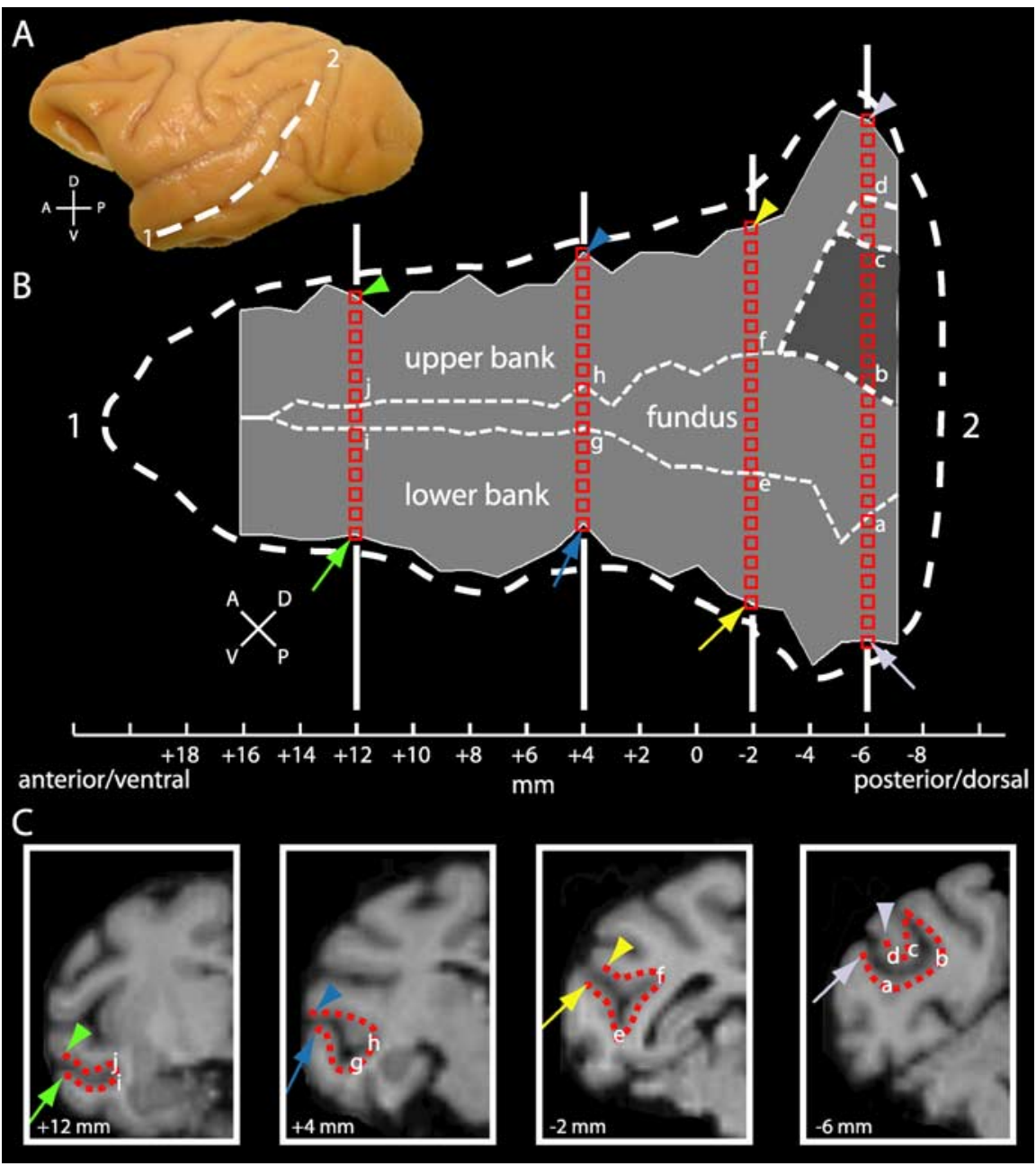

Figure 1. Flattened representation of the left STS. $\boldsymbol{A}$, Lateral view of the left hemisphere of a rhesus monkey. $\boldsymbol{B}$, Flattened STS possibly part of the parietal cortex. The flat map of the STS $(\boldsymbol{B})$ was made (for alignment, see Materials and Methods) by sampling along coronal sections of the template brain, as indicated in the four insets in $\mathbf{C}$. Coronal sections were sampled every millimeter m; red squares) starts near the lip of the lower bank (arrows), through the fundus, toward the lip of the upper bank (arrowheads). A, Anterior; D, dorsal; P, posterior; V, ventral.

Table 1. Fixation behavior in the different tests

\begin{tabular}{lccccc}
\hline Test & M1 & M3 & M4 & M5 & M6 \\
\hline Speed & 6 & & 10 & $15^{a}$ & \\
Opponent motion & 7 & 9 & & 12 & \\
Size & 14 & & & 7 & \\
Moving shapes & 15 & $6^{a}$ & & 22 & \\
Flow & 9 & 7 & & 15 & \\
Structure-from-motion & 12 & 13 & & $20^{a}$ & \\
Action & & 9 & & 10 & \\
Goal-directed action & 10 & $7^{a}$ & & 12 & \\
Action-moving object & & & & 11 & 10 \\
Eccentricity & & & & & \\
Adaptation & & & &
\end{tabular}

The number of saccades per minute is listed.

${ }^{a}$ Those tests in which that number differs significantly (one-way ANOVA) between conditions.

FST areas (Fig. 2, numbers 1, 2, and 3), in agreement with previous single-cell studies (Dubner and Zeki, 1971; Zeki, 1974; Ungerleider and Desimone, 1986a,b; Maunsell and Van Essen, 1987; Tanaka et al., 1993). Motion activation is also prominent on the 


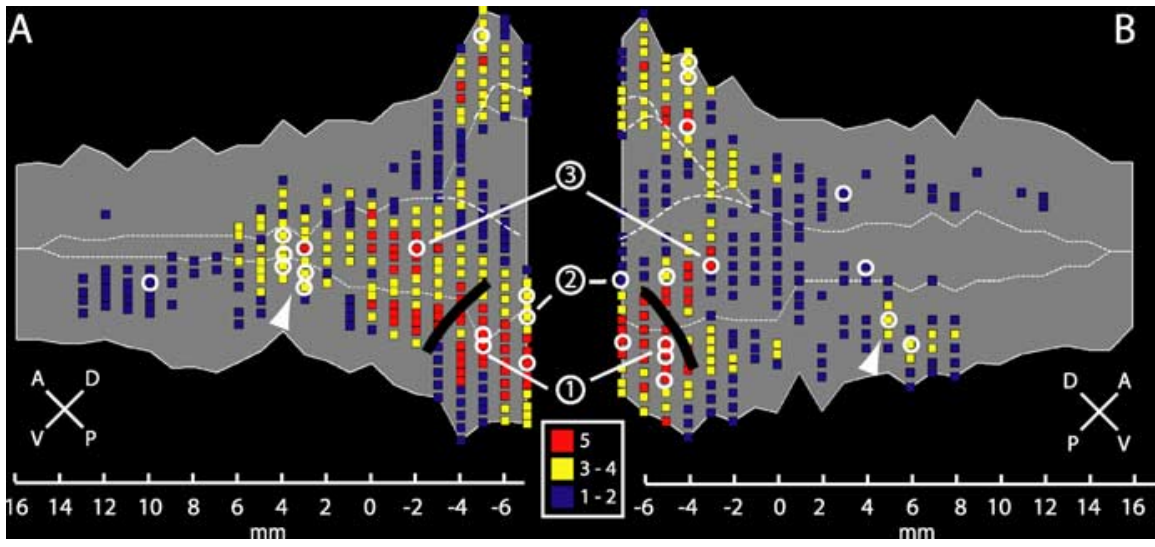

Figure 2. Motion-responsive regions within the STS. Maps of the flattened left $(\boldsymbol{A})$ and right $(\boldsymbol{B})$ STS show voxels responding significantly ( $p<0.05$, corrected for multiple comparisons) to moving stimuli (compared with static controls) in at least one (blue), at least three (yellow), or at least five (red) of the following subtractions: slowly moving (1\%s) RTPs compared with static control, fast moving $(16 \%$ s) RTPs compared with static control, expanding and contracting random dot patterns compared with static control, rotating random dot patterns compared with static control, and translating random dot patterns compared with static control. White circles indicate SPM local maxima. Numbers 1, 2, and 3 indicate local maxima of areas MT/V5, MSTV, and FST, respectively, following Vanduffel et al. (2001). White arrowheads indicate a motion-sensitive region in the middle of the lower bank of the STS. Data are derived from group analysis of three monkeys (M1, M4, and M5). A, Anterior; D, dorsal; P, posterior; V, ventral.

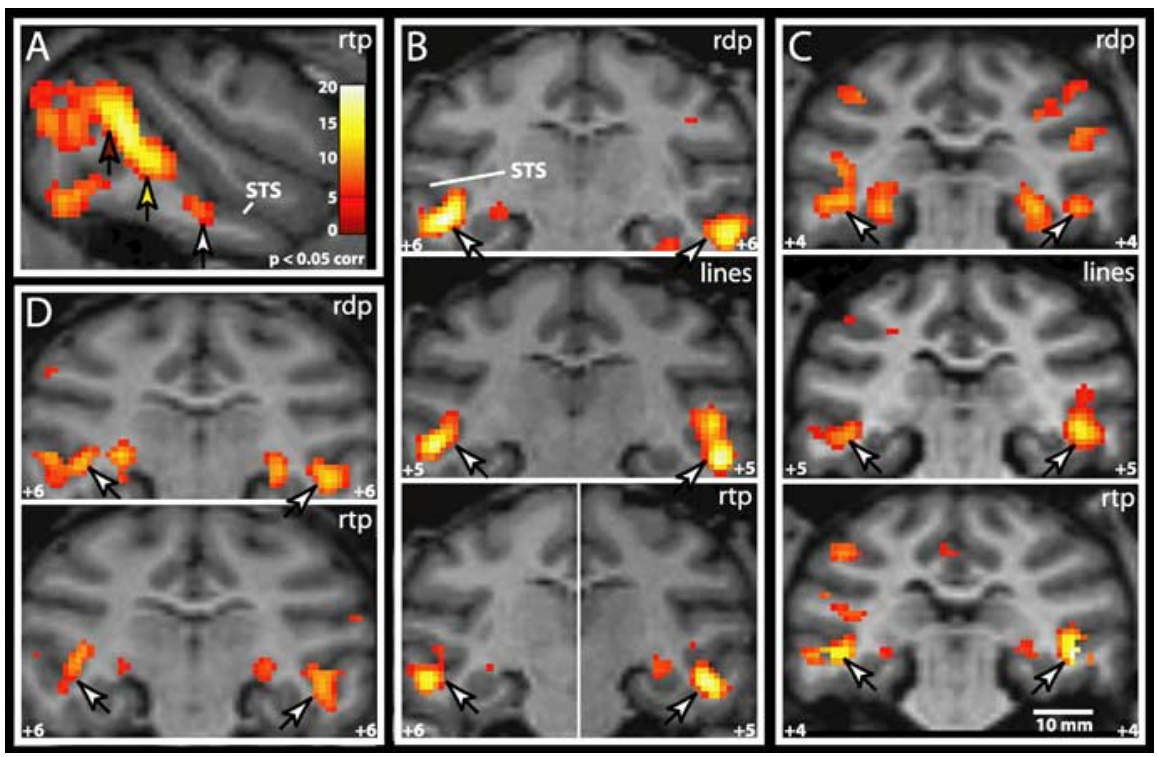

Figure 3. Location of the LST region in three monkeys. $\boldsymbol{A}$, Statistical map (M1) for moving versus stationary RTPs (rtp) superimposed onto a sagittal anatomical section of M3. Arrows indicate positions of MT/V5 (red), FST (yellow), and LST (white). $\boldsymbol{B}-\boldsymbol{D}$, Statistical maps for moving versus stationary RTPs (rtp), random dots (rdp), and random lines (lines) in M1 (B), M5 (C), and M4 (D). White arrows indicate the position of LST. All $t$ score maps are thresholded at $p<0.05$, corrected (corr) for multiple comparisons.

caudal upper bank corresponding to the MSTd location (Tanaka et al., 1986, 1993; Duffy and Wurtz, 1991; Graziano et al., 1994; Lagae et al., 1994). Note that anterior to the FST region, at the level of +3 to $+4 \mathrm{~mm}$ for the left hemisphere and +4 to $+6 \mathrm{~mm}$ for the right hemisphere, a consistent motion response was found in a region located in the lower bank and fundus of the STS (Fig. 2 , white arrowheads). Finally, the motion activation on the upper and lower bank at these anterior levels was clearly segregated in the right hemisphere but less so in the left hemisphere. The distinction between neighboring cortical regions exclusively by the localization of local maxima in the statistical landscape is difficult, even when maxima tend to cluster together in repeated tests (Fig. 2) and a contrast agent is used (see Materials and Methods).
The subsequent analysis, however, completely supports this initial identification.

To further explore the previously unknown motion-sensitive region revealed in the lower bank of the STS (Fig. 2, arrowheads), we tested motion sensitivity in the individual monkeys. As shown in Figure 3, motion activation consistently reached significance in this region in all three subjects (M1, M4, and M5) tested and for different types of motion stimuli: random dot, random textured, and random line patterns (Fig. 3, white arrows). This motion-sensitive region has been tentatively designated the LST region, in keeping with the nomenclature of its neighbors in the fundus (FST) and the medial part (MST) of the STS.

\section{Delineating motion-sensitive regions}

To separate the motion-responsive cortex into distinct functional regions, we focused on the results of four basic tests: the speed, optic flow, opponent motion grating, and shape tests (see Materials and Methods).

\section{Definition of the subtractions}

Single-cell studies have shown that the average optimal speed for MT/V5 neurons ranges from 8 to $32 \%$ s (Maunsell and Van Essen, 1983; Mikami et al., 1986; Lagae et al., 1993; Cheng et al., 1994). Thus, we contrasted the 8 and $1 \%$ s conditions in the speed test to reveal MT/V5. It is well established that many MSTd neurons are selective for optic flow components (Saito et al., 1986; Duffy and Wurtz, 1991; Graziano et al., 1994; Lagae et al., 1994). According to Tanaka et al. (1993), one of the defining properties distinguishing the MSTv area from the MSTd area is the nearly complete absence of cells selective for rotation and expansion/contraction in the MSTv area. Tanaka et al. (1993) reported that $18.1 \%$ of the direction-selective MSTd cells responded to expansion/contraction and $9.7 \%$ responded to rotation. In contrast, of the direction-selective cells in the MSTv area, only $1.3 \%$ were responsive to expansion/contraction and $1.4 \%$ were responsive to rotation. To localize the two subdivisions of the MST area, we chose the following two contrasts from the optic flow test: translation versus rotation to localize the MSTv area and expansion/contraction versus rotation to delineate the MSTd area. Furthermore, the optimal stripe width of a kinetic grating is assumed to reflect the RF size of a motion-sensitive neuron, because neurons with larger RFs are responsive to wider stripes (Van Doorn and Koenderink, 1982). Hence, we contrast the largest width $\left(4^{\circ}\right)$ with an intermediate width $\left(1^{\circ}\right)$ to isolate regions with large RFs, such as the MSTd (Raiguel et al., 1997) or STP (Bruce et al., 1981). Finally, we note that the motion-sensitive region tentatively labeled LST is located in the part of IT cortex sensitive to shape 


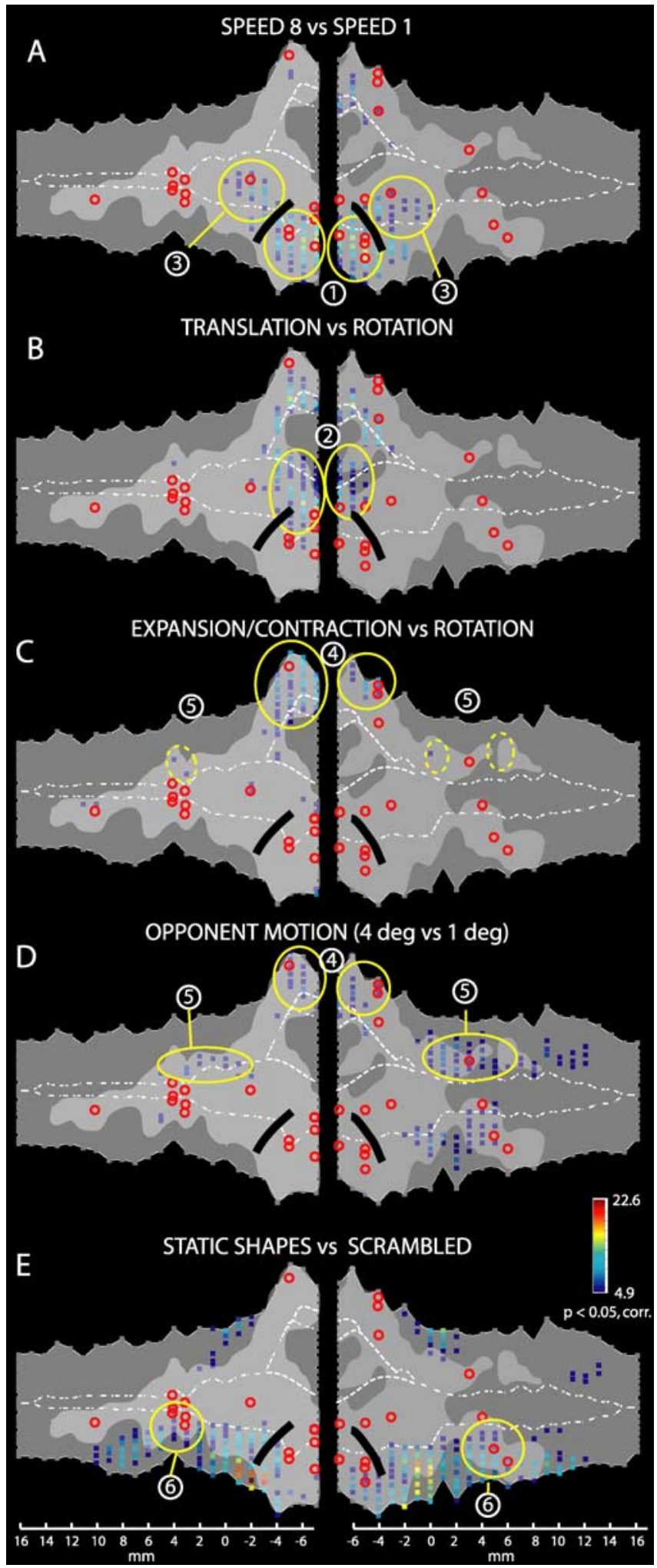

Figure 4. Definition and delineation of STS regions. Flat maps of the left and right STS show significant voxels $[p<0.05$, corrected (corr)] for several contrasts. $A$, Voxels responding significantly more to $8 \%$ compared with $1 \%$ s moving RTPs. Data from the group of three monkeys (M1, M4, and M5) are shown. $B$, C, Voxels responding significantly more to translating compared with rotating random dot patterns $(\boldsymbol{B})$ or expanding/contracting compared with rotating random dot patterns (C). Data from the group of three monkeys (M1, M4, and M5) are shown. $\boldsymbol{D}$, Voxels responding significantly more to kinetic gratings with wide stripes $\left(4^{\circ}\right)$ compared with similar kinetic gratings with intermediate stripe width $\left(1^{\circ}\right)$. Group data of two monkeys ( $(\mathrm{M} 1$ and M3) are shown. $\boldsymbol{E}$, Voxels corresponding to significantly higher responses to static grayscale
(Denys et al., 2004). Thus, we used the subtraction intact versus scrambled static shape from the moving shapes test to define LST as a region combining motion and shape sensitivity.

\section{Activation patterns and definition of the six motion-sensitive} regions

Figure 4 shows the voxels significant ( $p<0.05$, corrected) in the five subtractions. The light gray areas indicate the motionresponsive cortex defined as the voxels significant in at least one of the five motion contrasts of Figure 2. Red circles indicate the locations of the local maxima from these five contrasts. The black solid line corresponds to the vertical meridian (VM) between the MT/V5 and FST areas (Fize et al., 2003).

Area MT/V5 is known to be located in the lower bank of the caudal STS (Zeki, 1974; Maunsell and Van Essen, 1983, 1987; Desimone and Ungerleider, 1986). Contrasting RTPs moving at $8 \%$ vs $1 \%$ s revealed activity in the MT/V5 and in the FST areas located more anteriorly in the fundus (Fig. $4 A$, numbers 1 and 3 ), in agreement with our previous study (Vanduffel et al., 2001). Areas MT/V5 and FST (Fig. 5, pink and yellow outlines) are defined as all motion-sensitive voxels that were significant in the contrast $8 \%$ s versus $1 \%$ (Fig. $4 A$ ) and located, respectively, posterior and dorsal or anterior and ventral to the position of the VM.

Comparing translation to rotational motion activates mainly the fundus (Fig. $4 B$, number 2) and inner part of the upper bank of the STS, corresponding to the MSTv area, as described by Vanduffel et al. (2001). Contrasting expansion/contraction with rotation yielded an activation in the upper bank of the caudal STS (Fig. 4C, number 4), as expected from single-cell studies (Saito et al., 1986; Duffy and Wurtz, 1991; Graziano et al., 1994; Lagae et al., 1994). This identification is supported by contrasting wide $\left(4^{\circ}\right)$ and intermediate $\left(1^{\circ}\right)$ opponent motion stripe widths to map regions with large RFs (Fig. 4C,D, compare activation number 4). Thus, the MSTd area (Fig. 5, dark blue outline) was defined as all motion-sensitive voxels that were significant in either the contrast expansion/contraction versus rotation (Fig. 4C) or in the contrast wide versus middle motion stripe width (Fig. 4D). On the other hand, the MSTv area (Fig. 5, cyan outline) was defined as all motion-sensitive voxels significant in the subtraction translation versus rotation (Fig. $4 \mathrm{~B}$ ) but not already included in one the three aforementioned regions (MT/V5, FST, and MSTd). This latter restriction was required mainly in the left hemisphere to avoid overlap with the other regions. Notice that for symmetry, we included a few voxels into the right MSTv area that only reached the $p<0.001$ uncorrected threshold for motion sensitivity (Fig. 5, dark gray region within the cyan region).

We defined a motion sensitive region, STPm, located in the upper bank of the STS anterior to the MSTd area, using the spatial frequency and optic flow tests (Fig. 4C,D). Although the function of the STP region remains mostly unclear, it seems that at least part of this region is involved in the analysis of optic flow (Bruce et al., 1981; Anderson and Siegel, 1999). Yet, the contrast expansion/contraction to rotation yielded few significant voxels in the STP region (Fig. 4C). Because MR signals elicited by moving

images of objects compared with scrambled controls. Group data of two monkeys (M1 and M5) are shown. Light gray shaded regions correspond to motion-responsive regions as identified by the five contrasts of Figure 2. Red circles indicate SPM local maxima from those five motion contrasts. Circled regions correspond to areas MT/V5 (1), MSTv (2), FST (3), MSTd (4), STPm (5), and LST (6) (see Results). The black solid line indicates the location of the VM, which forms the border between areas MT/V5 and FST (Fize et al., 2003) 


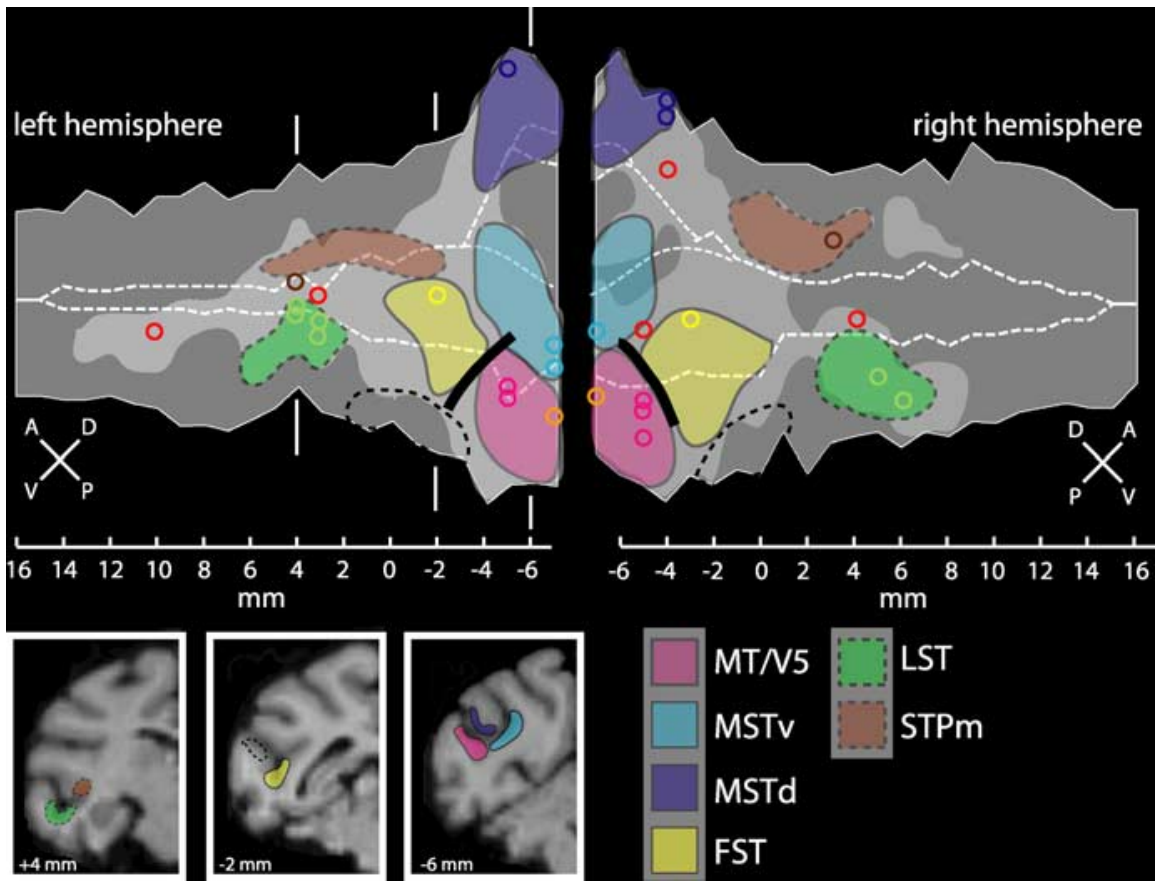

Figure 5. Overview of motion-responsive regions within the left and right STS. Boundaries of six motion-responsive regions are shown: MT/V5 (pink), MSTv (cyan), MSTd (blue), FST (yellow), STPm (brown), and LST (green). Boundaries that are less certain are indicated by dashed lines. The light gray overlay indicates the motion-responsive cortex identified with the five motion contrasts from Figure 2. Colored circles correspond to SPM local maxima from Figure 2. The black solid line indicates the location of the VM representation that forms the border between areas MT/V5 and FST. The dashed black line indicates the region giving strong responses to static shapes, probably corresponding to (part of) TE0. At the bottom, three coronal sections (at $-6,-2$, and $+4 \mathrm{~mm}$, respectively, to the interaural plane) show the locations of the different regions: MT/V5, MSTv, and MSTd at level -6 $\mathrm{mm}$; FST at $-2 \mathrm{~mm}$; and LST and STPm at $+4 \mathrm{~mm}$ to the interaural plane. A, Anterior; D, dorsal; P, posterior; $V$, ventral.

stimuli were weaker in the STP region than in the MSTd area (supplemental Fig. S2, available at www.jneurosci.org as supplemental material), we lowered the threshold from a $t$ score of 4.86 to 4.0 , corresponding to $p<0.0001$ uncorrected. This yielded a number of activated voxels in the middle portion of the upper bank of the STS, indicated by the circular yellow dashed lines at number 5 in Figure 4C. These portions of the STP region activated by the flow stimuli, also respond significantly $(p<0.05$, corrected) more to wide $\left(4^{\circ}\right)$ than to intermediate $\left(1^{\circ}\right)$ motion stripes (Fig. $4 D$, number 5 ). Activation was more extensive in the right hemisphere, extending more anteriorly in the upper bank and including the local maximum of motion sensitivity. Thus, the STPm region (Fig. 5, brown dashed outline) was defined as the motion-sensitive voxels anterior to the MSTd area that were significant in the contrast wide versus intermediate stripe widths or that reached a $t$ score of 4 in the contrast expansion/contraction versus rotation. This region is located on the upper bank $7-8 \mathrm{~mm}$ anterior to the MSTd area.

The LST region displayed some activation in the subtraction wide versus intermediate stripe width (Fig. 4D). A clearer definition was obtained by contrasting static shapes with their scrambled counterparts, which yielded significant activation along extended portions of the lower bank of the STS (Fig. 4E), in agreement with our previous study (Denys et al., 2004). The portion of this shape-sensitive region that is also motion sensitive corresponds to the LST (Fig. $4 E$, number 6). Thus, the LST (Fig. 5 , green dashed outline) was defined as those motion-sensitive voxels anterior to the FST area that were also shape sensitive. It is located in the lower bank of the STS, and its center lies $\sim 6-8 \mathrm{~mm}$ anterior to that of FST area. The region of the lower bank located between the FST and LST areas, which is shape but not motion sensitive (Fig. 5, black dashed outline), presumably corresponds to (part of) the temporal-occipital area (TEO) (Boussaoud et al., 1990).

Figure 5 gives a schematic overview in a flattened format of the six motionresponsive regions that we have delineated within the STS using the subtractions of Figure 4. Notice that these six motionsensitive regions include most of the local motion maxima defined in step 1 ( 12 of 14 and 11 of 14 in the left and right hemispheres, respectively). At the bottom of Figure 5, the locations of the six different motion-responsive regions are shown on three anatomical coronal sections. This can be compared with the motion activation patterns obtained in the speed test in monkey M1 (Fig. 6).

\section{Basic properties of motion-sensitive regions: retinotopy and direction adaptation \\ Retinotopic organization}

All five monkeys involved in the present study underwent our standard retinotopic tests: we contrasted the VM with the horizontal meridian, the central $3^{\circ}$ with the peripheral visual field (inner and outer diameters of 3 and $28^{\circ}$, respectively) and the upper with lower visual field as described by Fize et al. (2003). The testing of the projections of the meridians in the STS revealed no systematic representation of these meridians rostral to the VM representing the boundary between the FST and MT/V5 areas (Fize et al., 2003). Only a center-periphery organization was suggested for the FST area [Fize et al. (2003), their Fig. 6], in complete agreement with the results of Desimone and Ungerleider (1986). Indeed, these authors described a crude topographic organization in the MST and FST areas in which only the projections of the central and peripheral visual fields were segregated. Subsequent studies (Tanaka et al., 1993; Lagae et al., 1994) have emphasized the lack of retinotopic organization in the MST area.

The eccentricity test was introduced to examine this centerperiphery organization in the six motion-sensitive regions. Figure 7 shows the result of contrasting the centrally presented stimuli with the peripheral stimuli (main effects in both directions), yielding a representation of the central $2^{\circ}$ (red regions) and of the peripheral $7-14^{\circ}$ (blue regions). In MT/V5, the central and peripheral parts of the visual field are represented rostrally and caudally, respectively, in agreement with Fize et al. (2003) and with the organization described in the single-cell studies (Van Essen et al., 1981; Desimone and Ungerleider, 1986; Maunsell and Van Essen, 1987). The FST area also contains a segregated representation of the central and visual fields, with the central part abutting that of MT/V5, again in agreement with previous studies (Desimone and Ungerleider, 1986; Fize et al., 2003). MST areas represent mainly the peripheral visual field, in agreement with single-cell studies (Desimone and Ungerleider, 1986; Komatsu and Wurtz, 1988; Tanaka et al., 1993). The LST region includes mainly a central visual field representation, although the transition between the FST and LST areas represents the periph- 


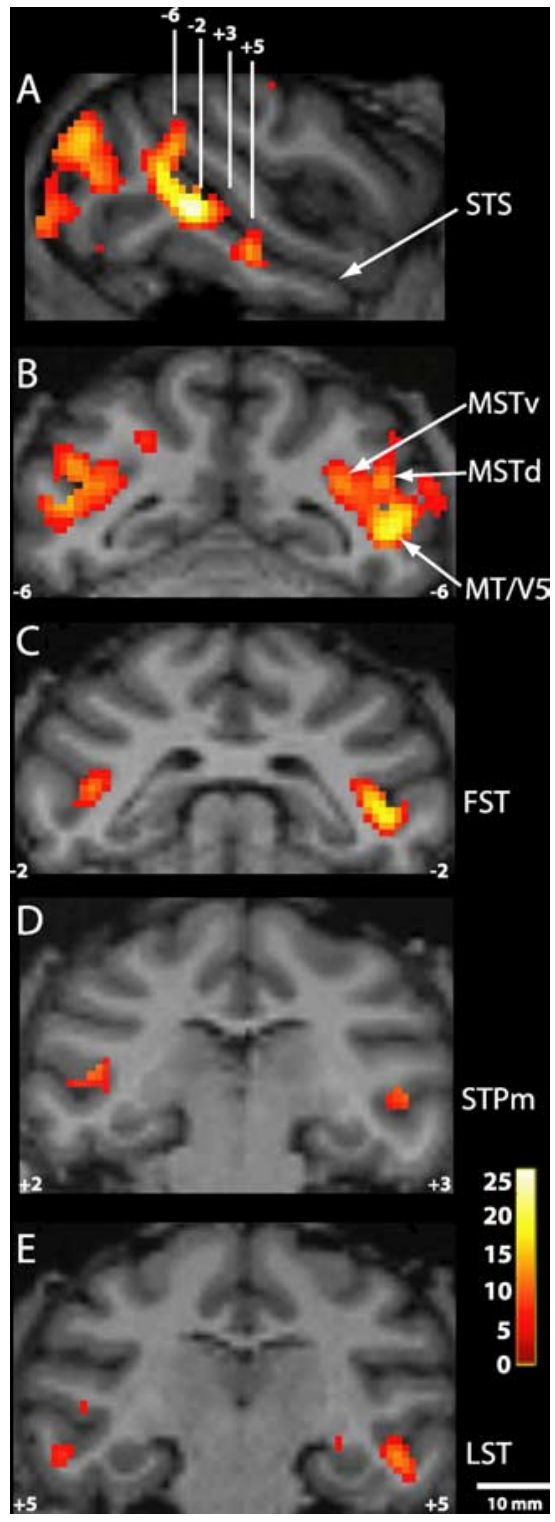

Figure 6. Motion-sensitive regions within the STS. Statistical parametric maps for moving compared with stationary RTPs in the left and right hemispheres of M1 (threshold $p<0.05$, corrected for multiple comparisons) are shown. $\boldsymbol{A}$, Sagittal section through the STS (arrow) indicating four different anteroposterior levels for which coronal sections are shown in $\boldsymbol{B}-\boldsymbol{E}$. $\boldsymbol{B}-\boldsymbol{E}$, Coronal sections showing the location of areas MT/V5, MSTv and MSTd (B), FST (C), STPm $(\boldsymbol{D})$, and LST $(\boldsymbol{E})$. The numbers indicate anteroposterior levels (slightly different for the right and left hemispheres in $\boldsymbol{D}$ ). The color scale indicates $t$ scores, thresholded at $p<0.05$, corrected for multiple comparisons.

ery. It is possible that we underestimate the caudal extent of LST, which represents the peripheral visual field. The topographic organization of the STPm area seems very crude, although there is some indication that its caudal part represents the peripheral visual field.

\section{Direction adaptation}

In single-cell studies, motion processing regions typically contain large proportions of direction-selective neurons. It has been proposed that directional adaptation in fMRI studies can provide an indirect indication of the presence of direction-selective neurons (Huk et al., 2001; Tolias et al., 2001). Single-cell studies agree that almost all MT/V5 neurons and most MST neurons are direction selective (Dubner and Zeki, 1971; Albright, 1984; Saito et al.,
1986; Tanaka et al., 1986; Duffy and Wurtz, 1991; Lagae et al., 1994). On the other hand, only one-third of the FST cells are direction selective for simple translation (Desimone and Ungerleider, 1986), and a fraction of the anterior STP neurons are also direction selective (Oram et al., 1993). Obviously, no such information is available for LST, and so we modified the direction adaptation paradigm of Huk et al. (2001) to provide indirect evidence on this issue. As shown in Figure 8, clear adaptation effects were observed in areas MT/V5, FST, and LST, whereas the effects in the STPm region were small. The difference between average MR signals in adapted and mixed blocks was significant (paired $t$ test, one-tailed, corrected for multiple comparisons) in areas MT/V5, FST, and LST (Fig. 8). In contrast, adaptation effects were absent in central V4 and V3A. These effects observed at the group level were also present in the single-subject analyses. Overall responses were weak in the MSTd and MSTv areas (Fig. 8 ), probably because of the restriction of the stimulus to the central $7^{\circ}$. Indeed, using a more peripheral stimulus yielded somewhat stronger responses in the MST areas and clear evidence for direction adaptation in these two areas, as well as in the STPm region (supplemental Fig. S3, available at www.jneurosci.org as supplemental material).

\section{Additional functional characteristics of well known STS motion-responsive regions}

To describe more fully the functional properties of the six motion regions of the STS, we statistically analyzed the complete set of tests, including the four basic tests used to define the motion regions and the additional tests. These tests probe the effects of lower-order motion parameters and of higher-order motion stimuli and their controls. We statistically tested the effects of stimulus conditions both within the regions (Tables 2, 3) and between regions (Table 4 ). These statistical analyses were performed on the percentage of signal change calculated for all voxels belonging to the six different ROIs as outlined in Figure 5. Because results were similar for the two hemispheres, data were pooled, except for the action tests. In these latter tests, the stimulation was asymmetric because the hand moved mainly in the right visual field; hence, we used only the ROIs of the left hemisphere. The mean and SEM (across all voxels of both hemispheres) for each condition and ROI are plotted in Figure 9 for the lower-order motion tests and the shape test and in Figure 10 for the higher-order motion tests. In describing the functional characteristics of the known or lesser known regions, we concentrate on those contrasts that were significant in the group and in at least two-thirds of the individual monkeys (Tables 2, 3).

\section{Area $M T / V 5$}

Figure $9 A$ shows the responses (relative to static baseline) of the six regions to the RTPs moving at different speeds $(1-16 \% \mathrm{~s})$. The largest MR signal changes were observed in MT/V5, followed by areas FST, LST, and MSTd. MR responses in MT/V5 increased significantly with speed (Table 2), and the strongest responses were observed for fast $(16 \%)$ moving stimuli. This is in agreement with single-cell studies showing that the average optimal speed for MT/V5 neurons ranges from 8 to $32 \%$ s (Maunsell and Van Essen, 1983; Mikami et al., 1986; Lagae et al., 1993; Cheng et al., 1994). Separate analysis of central and peripheral MT/V5 (data not shown), showed that peripheral MT/V5 was less responsive to slow speeds than central MT/V5 (Lagae et al., 1993). No condition in the opponent motion stripe width test (six widths plus transparent) produced a significantly different response in MT/V5 (Table 2, Fig. 9B). 


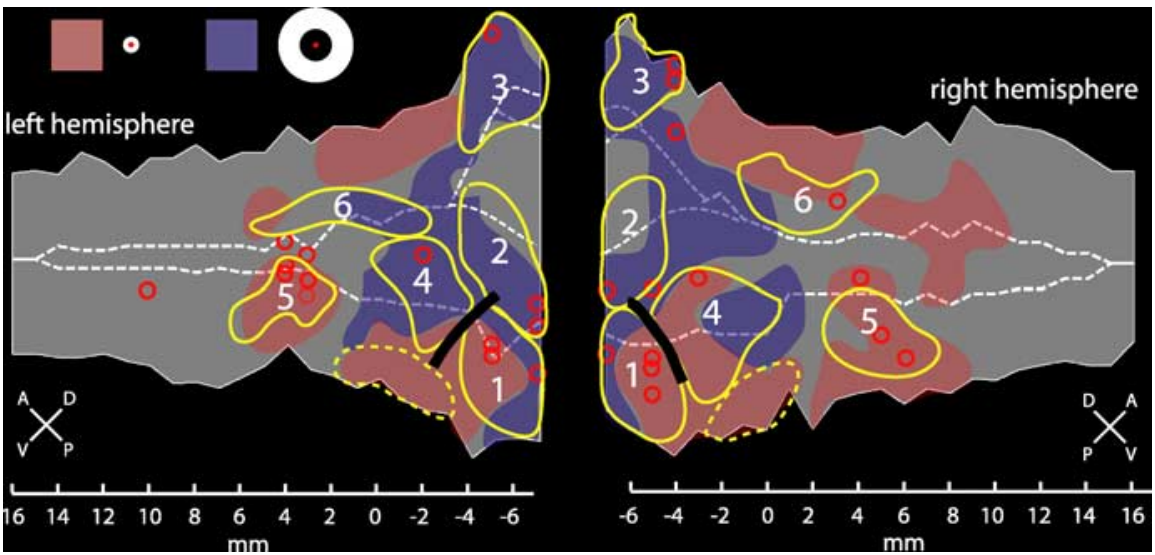

Figure 7. Eccentricity map of the STS. A flat map of the left and right STS shows regions responding significantly $(p<0.05$, corrected) to central $2^{\circ}$ stimulation (pink regions; compared with peripheral stimulation) and peripheral $7-14^{\circ}$ stimulation (blue regions; compared with central stimulation). Data are derived from group analysis of two monkeys (M1 and M5). Red circles indicate statistical parametric map local maxima from the five different motion contrasts in Fig. 2. The black solid line indicates the location of the VM, which forms the border between areas MT/V5 and FST (Fize et al., 2003). Yellow numbered outlines indicate the motion sensitive regions: 1, MT/V5; 2, MSTv; 3, MSTd; 4, FST; 5, LST; 6, STPm. Other conventions are as in Figure 2. A, Anterior; $D$, dorsal; $P$, posterior; $V$, ventral.

Area MT/V5 responds well to small $3^{\circ}$ diameter stimuli, but MR signals increase only modesty with increasing stimulus size (Fig. 9C; Table 2, see significant effect of conditions) (Bonferroni's post hoc test: 3 vs $7^{\circ}, p>1.0 ; 7$ vs $14^{\circ}, p<0.01 ; 14$ vs $28^{\circ}, p>$ $0.05)$. Most likely, the increased activation reflects the recruitment of additional neurons into the active population, offset to some extent by a decrease in responses to large stimuli in many of the individual neurons, as a consequence of the antagonistic surrounds demonstrated by $\sim 60 \%$ of the MT/V5 neurons (Tanaka et al., 1986; Raiguel et al., 1995).

In line with a previous study (Denys et al., 2004), MT/V5 showed no shape sensitivity (factorial ANOVA, main effect intact versus scrambled shapes not significant) (Table 3). Moving shapes and their scrambled moving controls evoke equal activation in MT/V5 (5.96 and 5.99\% MR signal change compared with fixation, respectively) (Fig. 9D). Static shapes and their scrambled controls also evoked very similar responses, but clearly smaller than those evoked by the motion conditions.

Compared with a static plus control condition, radial and circular motion evoked smaller (Table 2, see significant effect of conditions) (Bonferroni's post hoc test; $p<0.000001$ for both expansion/contraction vs translation and rotation vs translation) MR signal changes than translation in MT/V5 (Fig. 10A), in agreement with a previous single-cell study (Lagae et al., 1994) reporting that the average response to radial and circular motion was just over one-half the translation response.

Relative to uniform motion, transparent motion and kinetic shapes activate MT/V5 similarly (Table 2). Although individual MT/V5 neurons respond less to transparent motion than to uniform motion (Qian and Andersen, 1994), the MR signals in MT/V5 evoked by transparent motion exceeded those evoked by uniform motion (Fig. $10 \mathrm{~B}$ ). One possible explanation is that twice as many neurons are stimulated by patterns containing opposite directions of motion than by uniform motion, outweighing the reduced responses in the individual cells.

Random lines rotating in depth evoke significantly larger responses (Table 2) than translating random line stimuli in area MT/V5 (Fig. 10C), confirming Vanduffel et al. (2002). Finally, in the action tests, MT/V5 was significantly (Tables 2, 3) more active for the action conditions than the static and scrambled controls
(Fig. 10D,E). Furthermore, MT/V5 responded significantly more to action, compared with the static control, than to a small moving object, compared with its control (Table 3, Fig. 10 F).

\section{Medial superior temporal areas (MSTv and MSTd)}

Overall MR responses to moving RTPs (Fig. 9A-C) were weaker in the MSTd area compared with MT/V5, and even more so in the MSTv area. Like MT/V5, both areas MSTv and MSTd (Table 2, see significant effect of conditions for both areas) (Bonferroni's post hoc test; between $1 \%$ and $16 \%$; $p<0.0005)$ prefer faster $(16 \%)$ to slow $(1 \%$ s) speeds, in agreement with single-cell studies studies (Tanaka et al., 1989; Orban et al., 1995). Although both areas MSTv and MSTd (Table 2) showed a significant effect of conditions in the spatial frequency test (Fig. 9B), MR responses to gratings with wide $\left(4^{\circ}\right)$ versus narrow $\left(0.125^{\circ}\right)$ motion stripes were significantly different only in the MSTd area ( post hoc Bonferroni's test; $p<$ 0.001 for MSTd; $p>0.1$ for MSTv).

Areal summation was significant in both areas MSTv and MSTd (Table 2). It was somewhat stronger in the two MST regions (a factor of $\sim 3.3$ between 3 and $28^{\circ}$ ) compared with MT/V5 (factor of $\sim 1.5$ ) (Fig. 9C). For the MSTd area, this may be related to the smaller proportion of neurons exhibiting antagonistic surrounds (Tanaka et al., 1986; Lagae et al., 1994). For the MSTv area, one would predict less summation because one of the defining characteristics of the MSTv area is the responsiveness to small stimuli (Tanaka et al., 1993; Eikufu and Wurtz, 1999). However, the maximum diameter used in our study was only $28^{\circ}$ compared with $80^{\circ}$ in the study by Tanaka et al. (1993).

Both MSTv and MSTd areas (Table 1) failed to show any shape sensitivity (Fig. 9D). The main effect of motion was significant in both areas (Table 3), but only the MSTd area responded to both intact and scrambled moving shapes (compared with fixation baseline).

That the different optic flow conditions had a significant effect in the MSTv and MSTd areas (Table 2) follows from the definition of these areas (Fig. 4). Neither the MSTv nor the MSTd area was activated by kinetic shapes or transparent motion (compared with uniform motion), clearly setting them apart from MT/V5 (Fig. $10 \mathrm{~B}$ ). Furthermore, in strong contrast to MT/V5, both MST areas showed a preference for two-dimensional compared with three-dimensional motion displays (significant in the MSTV area) (Table 2, in italic; Fig. 10C). Also, action had little or no effect in the two MST areas (Fig. 10D-F).

\section{Additional functional characteristics of less known or unknown STS motion-responsive regions FST region}

MR responses to moving RTPs were stronger in the FST region than those in areas MSTd and MSTv (Fig. 9A-C), in agreement with previous studies (Vanduffel et al., 2001). One exception was the response to the random dot stimuli (Fig. 10 A) that was larger in the MSTd area than in the FST region (Table 4). MR responses to two- and three-dimensional moving random lines (Fig. 10C) were even slightly stronger in the FST region than in MT/V5 (Table 4). 
The FST region showed a significant preference for faster stimuli (Table 2, Fig. $9 A$ ), but the difference was less pronounced than in area MT/V5. Stripe width of kinetic gratings only had a modest effect in the FST region (Table 2). The lack of clear summation (Table 2 ) sets the FST region apart from MT/V5 and both MST regions (Fig. 9C).

The shape test (Fig. 9D) failed to reveal any significant shape response in the FST region (Table 3), in contrast to our previous study (Denys et al., 2004) in which larger static shapes were used.

Responses in the FST region to optic flow components differed (Table 2) and reflected the pattern observed in MT/V5 (Fig. 10A). More interestingly, the FST region seems to be involved in the analysis of opponent motion patterns, responding significantly better to kinetic shapes than to a transparent motion control stimulus (Fig. 10B, Table 2).

Consistent with previous fMRI studies (Sereno et al., 2002, 2005; Vanduffel et al., 2002), we observed that the FST region is involved in extracting three-dimensional structure from motion, because we observed significantly stronger responses to randomly connected lines rotating in depth compared with two-dimensional translating random lines (Table 2, Fig. 10C). Action had a significant effect in the FST region when compared with static and scrambled controls (Tables 2, 3). In fact, the activity pattern is similar to that of MT/ $\mathrm{V} 5$, except for a reduction in the response to the scrambled condition (Fig. 10D,E). As in MT/V5 (Fig. 10F), the interaction between the factors hand/object and nature of the stimulus (motion, static) was significant (Table 3)

\section{LST region}

Compared with areas MT/V5, MST, and FST (Fig. 9A), the LST region showed only a modest preference for faster speeds (Table 1), and the difference in response between extreme speeds was not significant (Bonferroni's post hoc test; between 1 and $16 \% s ; p>0.1$ ). Stripe widths of kinetic gratings had a significant effect in the LST region (Table 2, Fig. 9B), with responses that increased with stripe width. Like area FST, the LST region exhibited little spatial summation (Table 2, Fig. 9C).

By definition, the LST region showed strong shape responses, not only for static but also for moving grayscale images (significant main effect of shape) (Table 3, Fig. 9D). Although areas MT/V5 and FST respond to static stimuli just like the LST region (Figs. 9D, 10E), LST is the only region that displays shape sensitivity as evidenced by the significant effect of scrambling.

Optic flow conditions produced significantly different effects in the LST region (Table 2), and notably, rotation was as effective a stimulus as translation (Fig. 10A). Furthermore, the LST region responded to opponent motion patterns: responses to kinetic
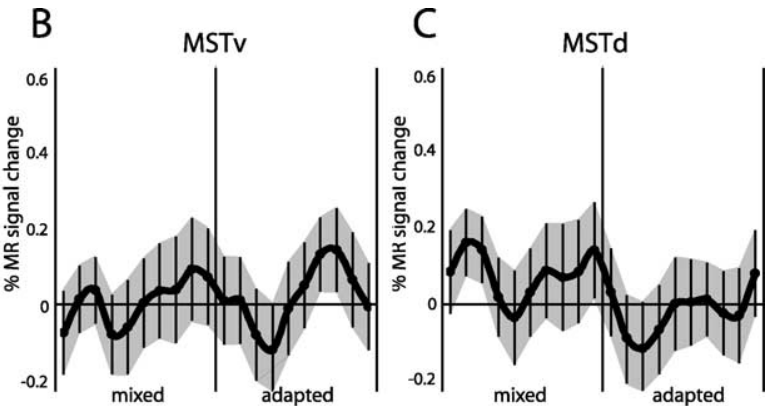

$\mathrm{E}$
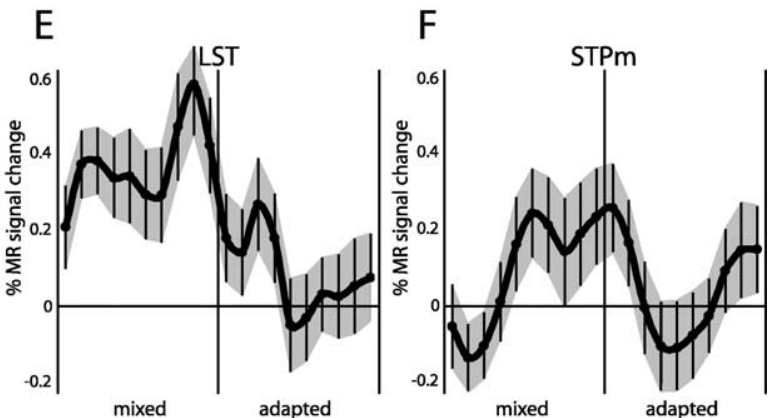

I
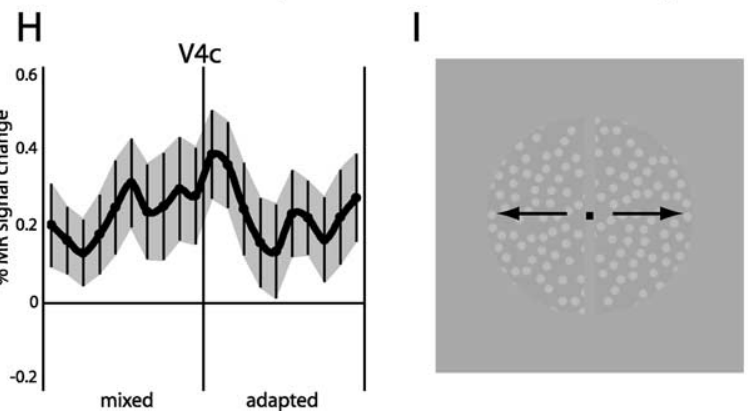

Figure 8. $\quad \boldsymbol{A}-\boldsymbol{H}$, Time course of MR signals (in percentage of change from fixation) in the mixed and adapted motion blocks of the direction adaptation test in areas MT/V5 (A), MSTv (B), MSTd $(\boldsymbol{C}), \operatorname{FST}(\boldsymbol{D}), \operatorname{LST}(\boldsymbol{E}), \operatorname{STPm}(\boldsymbol{F}), \mathrm{V} 3 \mathrm{~A}(\boldsymbol{G})$, and V4 central $(\boldsymbol{H})$. $\boldsymbol{I}$, The stimulus configuration. Vertical error bars indicate SEMs. The V3A and V4 (central $3^{\circ}$ ) ROls were derived from the retinotopic maps ., 2003) and included 138 and 335 voxels, respectively, for both hemispheres combined. Group data of two monkeys (MS avoid the transient after the adaptation block and the decrease in adaptation with time after the initial adaptation block. A mean MR signal was calculated per run for the adapted and the mixed blocks using the last eight values of each of the three middle blocks. Because we have a clear prediction from the direction selectivity of MT/V5 and MST neurons, these mean levels were compared in a one-tailed paired $t$ test, after correction for the number of comparisons (8 ROls). The difference in MR signals between mixed and adapted blocks was significant ( $p<0.05$ corrected) in areas MT/V5, FST, and LST but not areas MSTv, MSTd, STPm, V3A, or V4c. Results were similar in each of the individual monkeys.

shapes were significantly stronger than responses to transparent motion (Fig. 10B, Table 2). Unlike areas MT/V5 and FST, the LST region responded better to uniform than to transparent motion (Fig. 10B). The comparison of the MR responses to random lines rotating in depth versus translating in the fixation plane yielded little difference in the LST region (Table 2, Fig. 10C).

Finally, action had a significant effect in the LST region compared with static and scrambled controls (Tables 2, 3; Fig. $10 D, E)$. Unlike areas FST and MT/V5, the LST region is not responsive to the scrambled stimulus. Also, LST is the only region in which there is an interaction between the factor action and the factor presence/absence of the object (i.e., the goal), with the action effect being larger when the object is present. This effect was significant only in one of the two animals tested and can only be considered a tendency (Table 3). In the LST region, as in areas FST and MT/V5, the interaction between the factor hand/object and the dynamic nature of the stimuli (motion, static) was significant (Table 3). 
Table 2. Functional differences within regions (one-way ANOVA)

\begin{tabular}{|c|c|c|c|c|c|c|c|}
\hline \multirow[b]{2}{*}{ Test } & \multirow[b]{2}{*}{ Region } & \multirow[b]{2}{*}{ Group Fvalues } & \multicolumn{5}{|l|}{$p$ values } \\
\hline & & & Group & M1 & M3 & M4 & M5 \\
\hline \multirow[t]{6}{*}{ Speed (conditions: $1,2,4,8$, and $16^{\circ} / \mathrm{s}$ ) } & MT/V5 & $F_{(4,275)}=53.95$ & $\underline{0.000001}$ & 0.000001 & & 0.000001 & 0.000001 \\
\hline & MSTv & $F_{(4,250)}=5.68$ & 0.0002 & 0.005 & & 0.00015 & 0.000001 \\
\hline & MSTd & $F_{(4,225)}=6.19$ & 0.0001 & 0.000001 & & 0.005 & n.s. \\
\hline & FST & $F_{(4,255)}=21.54$ & 0.000001 & 0.000001 & & 0.000001 & 0.000001 \\
\hline & LST & $F_{(4,170)}=2.46$ & $\overline{0.047}$ & n.s. & & 0.00002 & n.s. \\
\hline & STPm & $F_{(4,170)}=3.47$ & 0.009 & n.s. & & n.s. & 0.000001 \\
\hline \multirow{6}{*}{$\begin{array}{l}\text { Spatial frequency (conditions: transparent } \\
\text { motion; } 4,2,1,0.5,0.25 \text {, and } 0.125 \text { cycles/ } \\
\text { degree) }\end{array}$} & MT/V5 & $F_{(6,385)}=1.61$ & n.s. & n.s. & 0.00006 & & \\
\hline & MSTv & $F_{(6,350)}=3.27$ & 0.004 & 0.009 & 0.0027 & & \\
\hline & MSTd & $F_{(6,315)}=5.76$ & 0.00001 & 0.0002 & 0.001 & & \\
\hline & FST & $F_{(6,357)}=3.37$ & 0.003 & n.s. & 0.00001 & & \\
\hline & LST & $F_{(6,238)}=12.09$ & $\underline{0.000001}$ & 0.000001 & 0.001 & & \\
\hline & STPm & $F_{(6,238)}=13.08$ & $\underline{0.000001}$ & 0.000001 & 0.00003 & & \\
\hline \multirow[t]{6}{*}{ Size (conditions: $3,7,14$, and $28^{\circ}$ ) } & MT/V5 & $F_{(3,220)}=15.37$ & 0.000001 & 0.000001 & & & 0.003 \\
\hline & MSTv & $F_{(3,200)}=28.82$ & 0.000001 & 0.000001 & & & 0.0027 \\
\hline & MSTd & $F_{(3,180)}=28.75$ & 0.000001 & 0.000001 & & & 0.000001 \\
\hline & FST & $F_{(3,204)}=1.22$ & n.s. & n.s. & & & 0.002 \\
\hline & LST & $F_{(3,136)}=0.74$ & n.s. & n.s. & & & n.s. \\
\hline & STPm & $F_{(3,136)}=0.72$ & n.s. & n.s. & & & 0.0002 \\
\hline \multirow{6}{*}{$\begin{array}{l}\text { Optic flow (conditions: expansion/contraction, } \\
\text { rotation, and translation) }\end{array}$} & MT/V5 & $F_{(2,165)}=58.54$ & 0.000001 & 0.000001 & & 0.000001 & n.s. \\
\hline & MSTv & $F_{(2,150)}=44.06$ & 0.000001 & 0.000001 & & 0.000001 & 0.000001 \\
\hline & MSTd & $F_{(2,135)}=13.50$ & 0.000001 & 0.0099 & & 0.0017 & 0.000001 \\
\hline & FST & $F_{(2,153)}=8.67$ & 0.00027 & n.s. & & 0.000001 & n.s. \\
\hline & LST & $F_{(2,102)}=10.652$ & 0.00006 & 0.002 & & 0.00004 & n.s. \\
\hline & STPm & $F_{(2,102)}=12.67$ & 0.00001 & 0.0018 & & 0.004 & 0.000001 \\
\hline \multirow{6}{*}{$\begin{array}{l}\text { Shape-from-motion (conditions: kinetic shape } \\
\text { and transparent motion) }\end{array}$} & MT/V5 & $F_{(1,110)}=2.76$ & n.s. & 0.000001 & 0.002 & & 0.000001 \\
\hline & MSTv & $F_{(1,100)}=8.53$ & 0.004 & 0.0001 & n.s. & & n.s. \\
\hline & MSTd & $F_{(1,90)}=1.56$ & n.s. & n.s & n.s. & & n.s. \\
\hline & FST & $F_{(1,102)}=42.34$ & 0.000001 & 0.006 & 0.000001 & & 0.000001 \\
\hline & LST & $F_{(1,68)}=213.01$ & $\underline{0.000001}$ & 0.000001 & 0.000001 & & 0.000001 \\
\hline & STPm & $F_{(1,68)}=107.71$ & 0.000001 & 0.0004 & 0.000001 & & 0.000001 \\
\hline \multirow{6}{*}{$\begin{array}{l}\text { Structure-from-motion (conditions: 2D trans } \\
\text { lation and 3D rotation-in-depth) }\end{array}$} & MT/V5 & $F_{(1,110)}=5.04$ & 0.027 & n.s. & 0.019 & & 0.002 \\
\hline & MSTv & $F_{(1,100)}=28.73$ & $\underline{0.000001}$ & 0.000001 & 0.000001 & & n.s. \\
\hline & MSTd & $F_{(1,90)}=9.68$ & 0.0025 & 0.000001 & n.s. & & n.s. \\
\hline & FST & $F_{(1,102)}=14.70$ & $\underline{0.00022}$ & n.s. & 0.0005 & & 0.000001 \\
\hline & LST & $F_{(1,68)}=0.11$ & n.s. & n.s. & n.s. & & n.s. \\
\hline & STPm & $F_{(1,68)}=3.97$ & n.s. & n.s. & 0.01 & & 0.035 \\
\hline \multirow{6}{*}{$\begin{array}{l}\text { Action (conditions: hand action, scramble, } \\
\text { and static) }\end{array}$} & MT/V5 & $F_{(2,87)}=28.69$ & $\underline{0.00001}$ & 0.00001 & 0.000001 & & 0.000001 \\
\hline & MSTv & $F_{(2,87)}=3.09$ & n.s. & n.s & n.s. & & 0.002 \\
\hline & MSTd & $F_{(2,87)}=15.014$ & 0.000001 & 0.001 & No resp. & & No resp. \\
\hline & FST & $F_{(2,66)}=122.62$ & $\underline{0.000001}$ & 0.000001 & 0.000001 & & 0.000001 \\
\hline & LST & $F_{(2,42)}^{(2,0)}=120.28$ & 0.000001 & 0.000001 & 0.0005 & & 0.000001 \\
\hline & STPm & $F_{(2,48)}^{(2,2)}=46.3$ & $\underline{0.000001}$ & 0.000001 & 0.000001 & & 0.000001 \\
\hline
\end{tabular}

One-way ANOVA for different tests (speed, flow, spatial frequency, shape-from-motion, size, and structure-from-motion) examining the effect of conditions (as indicated) within each of the six motion regions for the group and individua monkeys is shown. Bold indicates significance at $p<0.05$ in group, underlined indicates significance in group and in at least two-thirds of the subjects, and italics indicate significance for opposite contrast. n.s., Nonsignificant; No resp., MR response below the fixation control; $2 \mathrm{D}$, two-dimensional; $3 \mathrm{D}$, three-dimensional.

To control for possible differences in attention during presentation of the action and control stimuli, we performed a control experiment in which monkey M5 performed an acuity task (Vanduffel et al., 2001; Sawamura et al., 2005), whereas the action stimuli were presented in the background. The orientation of the bar changed to vertical at random moments, which the monkey had to indicate by a manual response. Despite a high performance $\left(98 \%\right.$ correct, 1923 trials) with a small $\left(0.13 \times 0.37^{\circ}\right)$ bar, results were very similar in the active and passive conditions (supplemental Fig. S4, available at www.jneurosci.org as supplemental material). In particular, the effect of the three conditions remained significantly different in the LST region, as in areas MT/V5, FST, and STPm, during the acuity task.

\section{STPm region}

Like most other regions, MR responses in the STPm region increased with increasing speed (Table 2, Fig. 9A). Stripe width of the kinetic gratings had a significant effect in the STPm region
(Table 2, Fig. 9B). Stimulus size had little effect on MR responses in the STPm region (Table 2, Fig. 9C).

The main effect of shape was significant in the STPm region (Fig. 9D, Table 3), but the difference between the responses to moving shapes and moving scrambled shapes was not significant in the STPm region (Bonferroni's post hoc test; $p>1.0$ ), in contrast to the LST region (Bonferroni's post hoc test; $p<0.00001$ ).

Optic flow responses in the STPm region were similar to those in area MSTd, although smaller in amplitude. STPm MR responses to optic flow components differed significantly (Table 2). The STPm region responded better to expansion/contraction compared with either rotation (Bonferroni's post hoc test; $p<$ 0.000001 ) or translation (Bonferroni's post hoc test; $p<0.05$ ) flow fields (Fig. 10A). Like area FST and the LST region, the STPm region responded significantly to shapes defined by motion (Fig. 10 B, Table 2). Like in the LST region, the response to two- or three-dimensional moving random lines was relatively 


\begin{tabular}{|c|c|c|c|c|c|}
\hline \multirow[b]{2}{*}{ Test } & \multirow[b]{2}{*}{ Region } & \multirow[b]{2}{*}{ Group $F$ values } & \multicolumn{3}{|l|}{$p$ values } \\
\hline & & & Group & M3 & M5 \\
\hline \multirow{22}{*}{$\begin{array}{l}\text { Moving shapes (conditions: moving shapes, } \\
\text { static shapes, moving scrambled, and static } \\
\text { scrambled) }\end{array}$} & MT/V5 & & & & \\
\hline & Main motion & $F_{(1,220)}=118.26$ & $\underline{0.000001}$ & 0.000001 & 0.000001 \\
\hline & Main shape & $F_{(1,220)}=0.47$ & $\overline{\text { n.s. }}$ & n.s. & n.s. \\
\hline & $\begin{array}{l}\text { Interaction } \\
\text { MSTV }\end{array}$ & $F_{(1,220)}=0.58$ & n.s. & n.s. & n.s. \\
\hline & Main motion & $F_{(1,200)}=90.53$ & 0.000001 & 0.000001 & n.s. \\
\hline & Main shape & $F_{(1,200)}=0.015$ & n.s. & n.s. & n.s. \\
\hline & $\begin{array}{l}\text { Interaction } \\
\text { MSTd }\end{array}$ & $F_{(1,200)}=0.32$ & n.s. & n.s. & n.s. \\
\hline & Main motion & $F_{(1,180)}=111.29$ & $\underline{0.000001}$ & 0.000001 & 0.000001 \\
\hline & Main shape & $F_{(1,180)}^{(1,180)}=0.20$ & n.s. & n.s. & n.s. \\
\hline & Interaction & $F_{(1,180)}^{(1,100)}=2.71$ & n.s. & n.s. & n.s. \\
\hline & FST & & & & \\
\hline & Main motion & $F_{(1,204)}=53.18$ & $\underline{0.000001}$ & 0.000001 & 0.000001 \\
\hline & Main shape & $F_{(1,204)}^{(1,24)}=1.58$ & n.s. & n.s. & 0.048 \\
\hline & Interaction & $F_{(1,204)}^{(1,204)}=0.10$ & n.s. & n.s. & n.s. \\
\hline & LST & & & & \\
\hline & Main motion & $F_{(1,136)}=132.67$ & $\underline{0.000001}$ & 0.000001 & 0.000001 \\
\hline & Main shape & $F_{(1,136)}^{(1,130)}=45.95$ & 0.000001 & 0.00006 & 0.000001 \\
\hline & Interaction & $F_{(1,136)}^{(1,130)}=0.86$ & n.s. & n.s. & n.s. \\
\hline & STPm & & & & \\
\hline & Main motion & $F_{(1,136)}=48.12$ & $\underline{0.000001}$ & 0.000001 & 0.000001 \\
\hline & Main shape & $F_{(1,136)}=7.93$ & 0.006 & 0.017 & 0.017 \\
\hline & Interaction & $F_{(1,136)}^{(1,10)}=1.15$ & $\overline{\text { n.s. }}$ & n.s. & n.s. \\
\hline \multirow{21}{*}{$\begin{array}{l}\text { Goal-directed action (conditions: action hand, } \\
\text { static hand, action mimic, and static mimic) }\end{array}$} & MT/V5 left & & & & \\
\hline & Main action & $F_{(1,116)}=101.86$ & $\underline{0.000001}$ & 0.000001 & 0.000001 \\
\hline & Main goal & $F_{(1,116)}=0.96$ & $\overline{\text { n.s. }}$ & n.s. & n.s. \\
\hline & $\begin{array}{l}\text { Interaction } \\
\text { MSTv left }\end{array}$ & $F_{(1,116)}=0.153$ & n.s. & n.s. & n.s. \\
\hline & Main action & $F_{(1,116)}=15.3$ & No resp. & No resp. & No resp. \\
\hline & Main goal & $F_{(1,116)}=0.67$ & n.s. & n.s. & n.s. \\
\hline & Interaction & $F_{(1,116)}=0.32$ & n.s. & n.s. & n.s. \\
\hline & $\begin{array}{l}\text { MSId left } \\
\text { Main action }\end{array}$ & $F_{(1116)}=80$ & No resp. & No resp. & No resp. \\
\hline & Main goal & $f_{(1,116)}^{(1,16)}=0.96$ & $\begin{array}{l}\text { n.s. } \\
\text { n. }\end{array}$ & n.s. & n.s. \\
\hline & Interaction & $F_{(1,116)}=0.24$ & n.s. & n.s. & n.s. \\
\hline & FST left & & & & \\
\hline & Main action & $F_{(1,88)}=80.1$ & $\underline{0.000001}$ & 0.000001 & 0.000001 \\
\hline & Main goal & $F_{(1,88)}=0.90$ & n.s. & n.s. & n.s. \\
\hline & $\begin{array}{l}\text { Interaction } \\
\text { LST left }\end{array}$ & $F_{(1,88)}=0.20$ & n.s. & n.s. & n.s. \\
\hline & Main action & $F_{(1,56)}=16.96$ & $\underline{0.0001}$ & 0.0004 & 0.006 \\
\hline & Main goal & $F_{(1,56)}=2.90$ & n.s. & n.s. & n.s. \\
\hline & Interaction & $F_{(1,56)}^{(1,50)}=4.02$ & 0.0498 & 0.01 & n.s. \\
\hline & STPm left & & & & \\
\hline & Main action & $F_{(1,64)}=96.42$ & $\underline{0.000001}$ & 0.000001 & 0.000001 \\
\hline & Main goal & $F_{(1,64)}=0.20$ & n.s. & n.s. & n.s. \\
\hline & Interaction & $F_{(1,64)}=0.26$ & n.s. & n.s. & n.s. \\
\hline \multirow{21}{*}{$\begin{array}{l}\text { Action-moving object (conditions: action hand, } \\
\text { static hand, moving object, and static object) }\end{array}$} & MT/V5 left & & & & \\
\hline & Main hand & $F_{(1,116)}=60.75$ & 0.000001 & 0.00001 & 0.000001 \\
\hline & Main motion & $F_{(1,116)}=74.44$ & 0.000001 & 0.000001 & 0.000001 \\
\hline & $\begin{array}{l}\text { Interaction } \\
\text { MSTy }\end{array}$ & $F_{(1,116)}=23.23$ & 0.000001 & 0.001 & 0.000001 \\
\hline & Main hand & $F_{(1,116)}=0.0005$ & n.s. & n.s. & \\
\hline & Main motion & $F_{(1,116)}=2.01$ & n.s. & n.s. & No resp. \\
\hline & Interaction & $F_{(1,116)}=3.93$ & n.s. & n.s. & No resp. \\
\hline & MSTd left & & & & Neresn \\
\hline & $\begin{array}{l}\text { Main hand } \\
\text { Main motion }\end{array}$ & $\begin{array}{l}F_{(1,116)}=3.58 \\
F_{(1116)}=26.44\end{array}$ & $\begin{array}{l}\text { n.s. } \\
\text { No resp. }\end{array}$ & $\begin{array}{l}\text { n.s. } \\
\text { No resp. }\end{array}$ & $\begin{array}{l}\text { No resp. } \\
\text { No resp. }\end{array}$ \\
\hline & Interaction & $F_{(1,116)}=20.41$ & No resp. & No resp. & 0.001 \\
\hline & FST left & & & & \\
\hline & Main hand & $F_{(1,88)}=20.99$ & 0.00002 & 0.0007 & 0.000001 \\
\hline & Main motion & $F_{(1,88)}=65.93$ & 0.000001 & 0.000001 & 0.000001 \\
\hline & $\begin{array}{l}\text { Interaction } \\
\text {. }\end{array}$ & $F_{(1,88)}=9.82$ & $\overline{0.002}$ & 0.0035 & 0.0035 \\
\hline & Mainhand & $F=19.45$ & 0.00005 & ns & 0.000001 \\
\hline & Main motion & $F_{(1,56)}^{(1,56)}=58.07$ & 0.000001 & 0.0002 & 0.000001 \\
\hline & Interaction & $F_{(1,56)}=9.72$ & 0.003 & 0.043 & 0.0004 \\
\hline & STPm left & & & & \\
\hline & Main hand & $F_{(1,64)}=1.40$ & n.s. & & 0.028 \\
\hline & Main motion & $F_{(1,64)}=160.41$ & 0.000001 & 0.000001 & 0.000001 \\
\hline & Interaction & $F_{(1,64)}=32.06$ & 0.000001 & 0.00001 & 0.046 \\
\hline
\end{tabular}


Table 4. Functional differences between regions

\begin{tabular}{|c|c|c|c|c|c|c|c|}
\hline \multirow[b]{2}{*}{ Test } & \multirow[b]{2}{*}{ Regions } & \multirow[b]{2}{*}{ Group Fvalues } & \multicolumn{5}{|l|}{$p$ values } \\
\hline & & & Group & M1 & M3 & M4 & M5 \\
\hline \multicolumn{8}{|l|}{ Speed (1 vs $16^{\circ} / \mathrm{s}$ ) } \\
\hline & MT/V5 and FST & $F_{(1,212)}=19.88$ & 0.00001 & 0.00028 & & 0.03 & 0.03 \\
\hline & MT/V5 and MSTv & $F_{(1,210)}=76.56$ & 0.000001 & 0.000001 & & 0.00003 & 0.000001 \\
\hline & MSTv and MSTd & $F_{(1,190)}=6.66$ & 0.01 & 0.004 & & n.s. & 0.038 \\
\hline & MSTv and FST & $F_{(1,202)}=19.84$ & $\overline{0.00001}$ & n.s. & & 0.04 & 0.000001 \\
\hline & FST and LST & $F_{(1,170)}=25.20$ & 0.000001 & 0.00009 & & n.s. & 0.000001 \\
\hline \multicolumn{8}{|l|}{ Size (3 vs $\left.28^{\circ}\right)$} \\
\hline & MT/V5 and FST & $F_{(1,212)}=11.43$ & 0.00086 & 0.002 & & & n.s. \\
\hline & MT/V5 and MSTv & $F_{(1,210)}=4.85$ & 0.028 & n.s. & & & 0.008 \\
\hline & MSTv and FST & $F_{(1,202)}=4.19$ & $\underline{0.042}$ & 0.011 & & & 0.038 \\
\hline & MSTV and MSTd & $F_{(1,190)}=8.13$ & 0.00485 & 0.015 & & & 0.000001 \\
\hline & MSTd and STPm & $F_{(1,158)}=25.43$ & $\overline{0.000001}$ & 0.00002 & & & 0.000001 \\
\hline \multicolumn{8}{|l|}{ Moving shapes } \\
\hline \multirow[t]{3}{*}{ Main-effect motion } & MT/V5 and MSTv & $F_{(1,210)}=78.9$ & $\underline{0.000001}$ & & 0.000001 & & 0.000001 \\
\hline & MSTv and MSTd & $F_{(1,380)}=43.63$ & 0.000001 & & 0.000001 & & 0.0003 \\
\hline & LST and STPm & $F_{(1,272)}=24.77$ & 0.000001 & & 0.0014 & & 0.000001 \\
\hline \multicolumn{8}{|l|}{ Optic flow } \\
\hline \multirow[t]{2}{*}{ ExCon vs Transl } & MSTv and MSTd & $F_{(1,190)}=4.93$ & 0.027 & n.s. & & 0.0061 & n.s. \\
\hline & LST and STPm & $F_{(1,136)}=26.78$ & 0.000001 & 0.0001 & & 0.000001 & 0,016 \\
\hline \multirow[t]{4}{*}{ ExCon vs Rot } & MT/V5 and MSTv & $F_{(1,210)}=28.48$ & 0.000001 & 0.00001 & & 0.00007 & n.s. \\
\hline & MSTv and MSTd & $F_{(1,190)}=9.49$ & $\underline{0.002}$ & 0.014 & & 0.0061 & n.s. \\
\hline & MSTv and FST & $F_{(1,202)}=7.25$ & $\overline{0.008}$ & 0.02 & & 0.0018 & n.s. \\
\hline & LST and STPm & $F_{(1,136)}=47.7$ & 0.000001 & 0.000001 & & 0.000001 & n.s. \\
\hline \multicolumn{8}{|l|}{ Shape-from-motion } \\
\hline \multirow[t]{5}{*}{ KinSha versus Transp } & MT/V5 and FST & $F_{(1,212)}=18.82$ & 0.00002 & 0.000001 & n.s. & & 0.001 \\
\hline & FST and LST & $F_{(1,170)}=20.83$ & 0.00001 & 0.000001 & n.s. & & 0.00003 \\
\hline & LST and STPm & $F_{(1,136)}=42.83$ & 0.000001 & 0.000001 & n.s. & & 0.006 \\
\hline & MSTv and FST & $F_{(1,202)}=50.20$ & $\underline{0.000001}$ & n.s. & 0.000001 & & 0.000001 \\
\hline & MSTd and STPm & $F_{(1,158)}=40.98$ & 0.000001 & 0.000001 & 0.000001 & & 0.009 \\
\hline \multicolumn{8}{|l|}{ Structure-from-motion } \\
\hline \multirow[t]{4}{*}{ 3D-Rot versus 2D-Transl } & MT/V5 and MSTv & $F_{(1,210)}=15.15$ & 0.00013 & 0.005 & 0.00002 & & 0.001 \\
\hline & MSTv and FST & $F_{(1,202)}=31.36$ & 0.000001 & 0.004 & 0.000001 & & 0.000001 \\
\hline & FST and LST & $F_{(1,170)}=5.13$ & $\underline{0.025}$ & n.s. & 0.004 & & 0.005 \\
\hline & MSTd and STPm & $F_{(1,158)}=11.99$ & 0.0007 & 0.014 & 0.013 & & 0.035 \\
\hline \multicolumn{8}{|l|}{ Goal-directed action } \\
\hline \multirow[t]{5}{*}{ Main effect action } & MT/V5 and MSTv & $F_{(1,232)}=43.44$ & $\underline{0.000001}$ & & 0.001 & & 0.000001 \\
\hline & FST and LST & $F_{(1,144)}=19.29$ & 0.000001 & & 0.00001 & & 0.0002 \\
\hline & LST and STPm & $F_{(1,120)}=10.69$ & 0.001 & & 0.000001 & & n.s. \\
\hline & MSTd and STPm & $F_{(1,180)}=16.31$ & 0.00008 & & 0.0001 & & n.s. \\
\hline & MSTv and FST & $F_{(1,204)}=49.99$ & $\underline{0.000001}$ & & 0.000001 & & 0.000001 \\
\hline \multirow[t]{3}{*}{ Mimic vs static } & FST and LST & $F_{(1,72)}=18.89$ & $\underline{0.00004}$ & & 0.00001 & & 0.0007 \\
\hline & LST and STPm & $F_{(1,60)}=9.16$ & 0.0036 & & 0.00005 & & n.s. \\
\hline & MSTd and STPm & $F_{(1,90)}=6.11$ & 0.015 & & 0.022 & & n.s. \\
\hline
\end{tabular}

Factorial ANOVA testing interactions between factors condition (left column) and region (second column), assessing differences between neighboring regions is shown. Results for group and individual monkeys are listed. Bold indicates significance at $p<0.05$ in group, and underlined indicates significance in group and in at least two-thirds of the subjects. n.s., Nonsignificant; ExCon, expansion/contraction; Transl, translation; Rot, rotation; KinSha, kinetic shape;

Transp, transparent; 3D-Rot, three-dimensional rotation; 2D-Transl, two-dimensional translation.

similar (Table 2, Fig. 10C). Action, however, had a significant effect compared with static and scrambled controls (Tables 2, 3; Fig. $10 D, E)$. In fact, the activity patterns were relatively similar in the STPm and LST regions (Fig. 10D-F), except that the STPm region does not respond to static stimuli and the LST region showed a tendency to be more strongly activated by goal-directed than mimicked actions (relative to static controls), whereas the presence of a goal (object) made no difference in the STPm region (Table 3).

\section{Functional differences between neighboring regions}

The functional description of the six motion-sensitive STS regions provided by Figures 9 and 10 and Tables 2 and 3 suggests that these regions differ in a number of functional properties. To validate the parcellation shown in Figure 5, we statistically evaluated the differences in functional properties between neighboring regions. We used a two-way ANOVA with regions and con- ditions as factors and list the interactions in Table 4. At least three functional properties differed between each of the pairs of neighboring regions. Speed sensitivity (responses to 1 and 16\%s) and responses to kinetic shapes relative to transparent control differentiate MT/V5 from area FST. These two areas are also separated by a representation of the VM (Fize et al., 2003). Areas MT/V5 and MSTv differ in sensitivity to speed, optic flow, and threedimensional structure from motion, as well as in their main effect of motion in the moving shape test and of action in the action test. The MSTv area differs from the FST area in sensitivity to speed, optic flow, three-dimensional structure from motion and action, as well as in spatial summation. Areas MSTv and MSTd differ in speed sensitivity, spatial summation, and relative response to radial and circular motion, as well as in their main effect of motion in the moving shape test. Area MSTd differs from the STPm region in spatial summation, in sensitivity to three-dimensional structure from motion and in response to kinetic shapes relative 

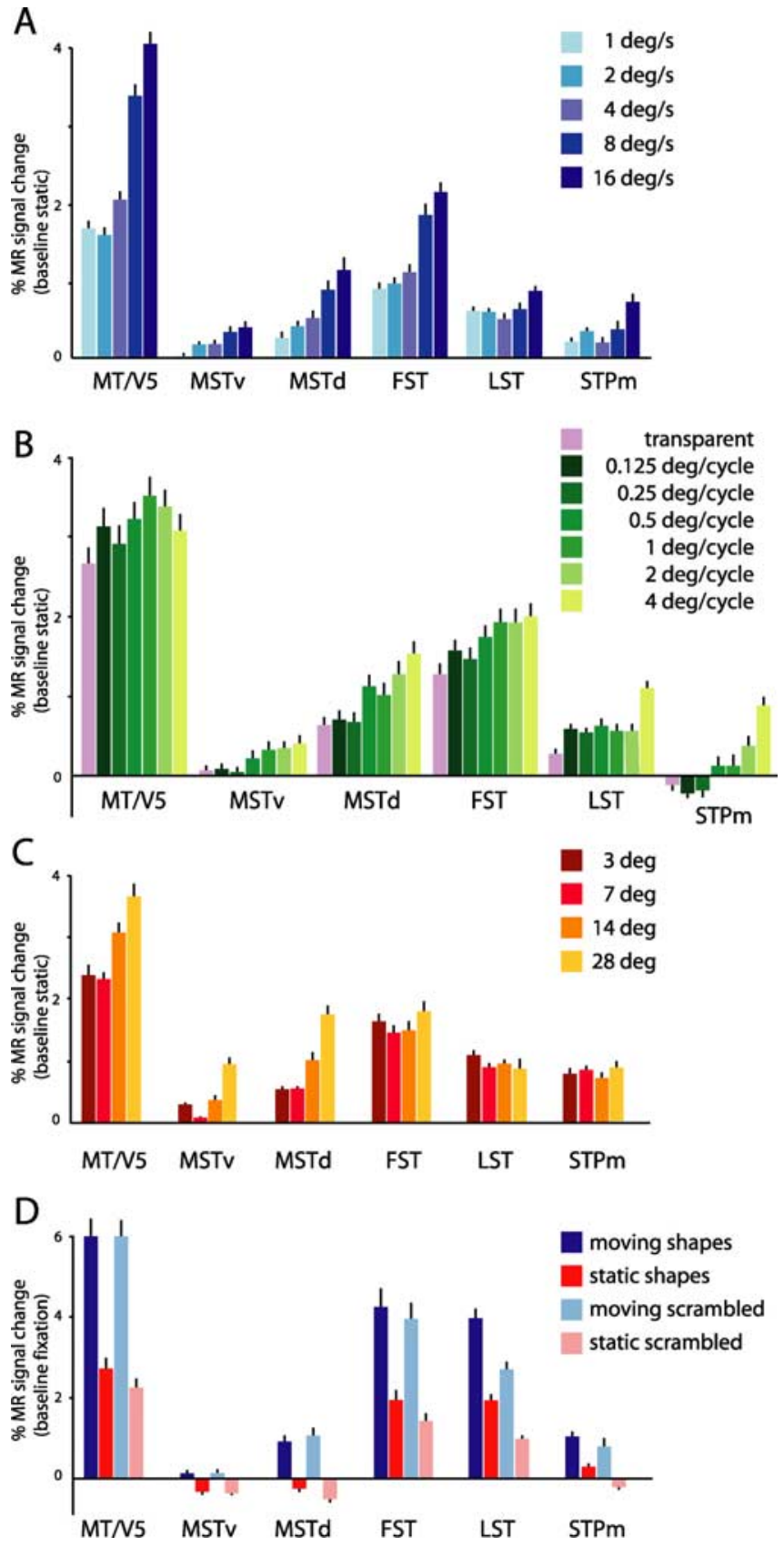

Figure 9. MR response profiles of the six STS motion regions: translation and shape tests. $A$, Percentage of MR signal changes for moving (speeds: 1, 2, 4, 8, and 16\%) RTPs compared with static control. Group data of three monkeys (M1, M4, and M5) are shown. B, Percentage of MR signal changes for transparent motion and kinetic gratings with different stripe widths $(0.125-$ $4^{\circ}$ ) compared with static control. Group data of two monkeys (M1 and M3) are shown. C, Percentage of MR signal changes for moving RTPs (compared with static control) varying in size $\left(3,7,14\right.$, and $28^{\circ}$ diameter). Group data of two monkeys (M1 and M5) are shown. $\boldsymbol{D}$, Percentage of MR signal changes for moving (red) and static (dark blue) shapes and for moving (pink) and static (light blue) scrambled shapes. Baseline is a fixation-only condition. Group data of two monkeys (M1 and M5) are shown. The percentage of MR signal changes were calculated for all voxels within the colored regions of Figure 5. Error bars indicate variability (SEMs) between voxels. deg, Degree.

to transparent motion. Area FST differs from the LST region in sensitivity to speed and three-dimensional structure from motion, as well as in response to kinetic shapes relative to transparent motion. Both the main effect of action and the interaction between the factors action and presence of a goal also differenti- ate between FST and LST. The LST region differs from STPm in relative response to radial motion and translation or circular motion and in its relative response to kinetic shapes. It is important to note that for each pair of regions at least one functional property does not belong to those used to define the six regions and thus constitutes an a posteriori validation.

It has been well documented that the hemodynamic response function (HRF) differs between cortical regions, although it is unclear how much of these differences reflect differences in neuronal properties or differences in vasculature (Schacter et al., 1997; Buckner et al., 1998; Robson et al., 1998; Miezin et al., 2000; Handwerker et al., 2004). For the present study, in which we attempt to demonstrate differences in neuronal properties between neighboring regions, the differences in vasculature are critical. Although there is some evidence for regional inhomogeneity at the venous level, critical for the BOLD signal (Robson et al., 1998), we know of little such evidence regarding the arterial side that underlies the blood volume-based methods as used here (see Materials and Methods). Furthermore, for block designs, only the differences in amplitude of the HRF would mater. Because it is unknown whether HRF amplitude can differ between cortical regions for blood volume-based MR signals and because ANOVA is a linear technique that does not correct for differences in gains of the MR response, we performed an additional analysis, suggested by one of the anonymous reviewers. All MR signals shown in Figures 9 and 10 were normalized by dividing them by the average percentage of MR signal change, calculated between all conditions for a given region as shown in these two figures. These normalized MR signals were then subjected to a two-way ANOVA (regions and conditions as factors) as before. As shown in Table S1 (available at www.jneurosci.org as supplemental material), the resulting interactions are very similar to those obtained without normalization (Table 4). Only 4 of the 29 differences between regions were removed by normalization, and two of these involved the two parts of area MST.

\section{Functional differences occur at borders between areas}

Table 4 indicates that for most pairs of neighboring regions, the average MR activity in at least three tests differs significantly between the elements of the pair. The final step for validating the parcellation is to show that such changes occur steeply at the border between the two regions. This is demonstrated by plotting MR activity along the length axis of the STS (Fig. 11) or across both banks of the STS (Fig. 12). Along the length axis of the lower bank, the plot crosses from MT/V5 into area FST and from area FST into the LST region. The speed sensitivity and the relative response to kinetic shapes compared with transparent change at both borders, most clearly at the MT/V5-FST border (Fig. $11 A, B)$. On the other hand, the sensitivity to three-dimensional structure from motion vanishes at the FST-LST border (Fig. $11 C)$. At that boundary, the difference between mimicked action and its static control also disappears (Fig. 11D).

Coronal sections show the changes encountered when crossing from MT/V5 into areas MSTv and MSTd (Fig. 12). Although area MSTv responds equally well to circular and radial flow, these two optic flow components evoked different but opposite responses in areas MT/V5 and MSTd (Fig. 12A). Whereas the main effect of motion in the shape test (difference between blue and red curves) is small in area MSTv, it is large in area MSTd and even more so in MT/V5 (Fig. 12B). Finally, the relative sensitivity to three-dimensional structure from motion and its twodimensional control reverses when crossing from MT/V5 into area MSTv (Fig. 12C). Coronal sections (Fig. 12D-G) also indi- 

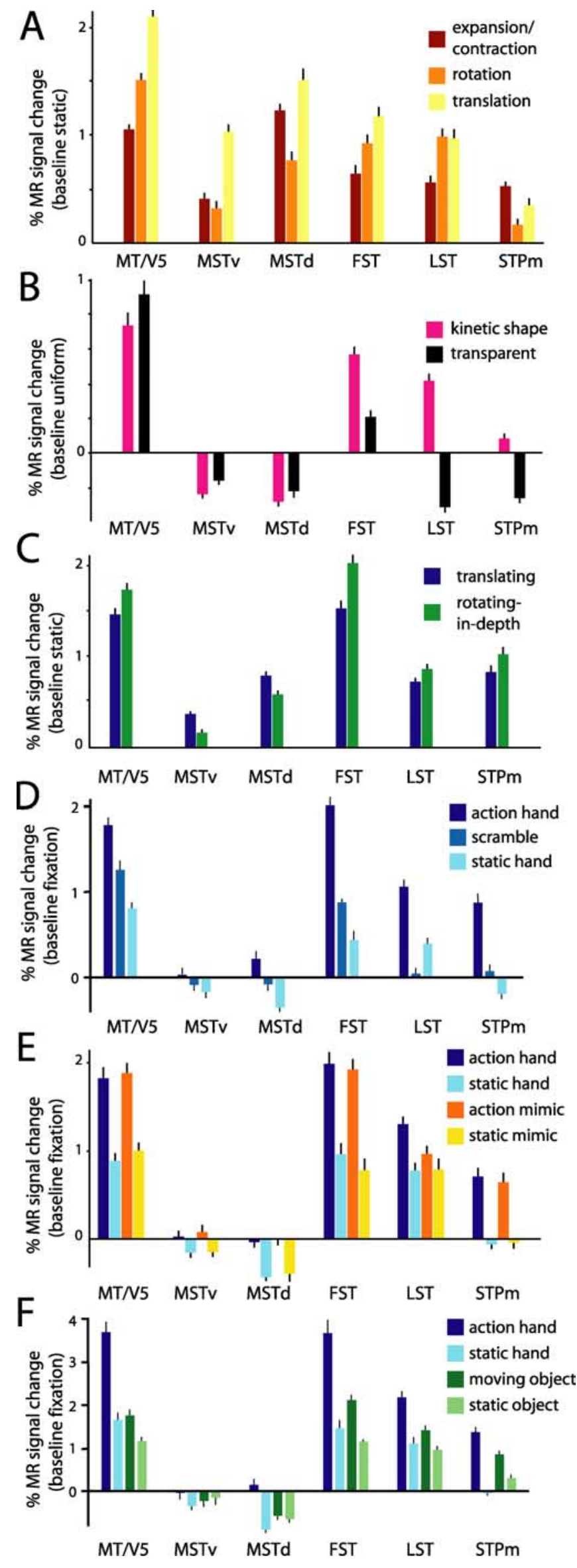
Percentage of MR signal changes for expanding/contracting (red), rotating (orange), and translating (yellow) random dot patterns (compared with static plus control). Group data of three monkeys (M1, M4, and M5) are shown. B, Percentage of MR signal changes for shapes defined by motion (kinetic shapes) and transparent motion compared with uniform motion (see Materials and Methods). Group data of three monkeys (M1, M3, and M5) are shown. C, Percentage of MR signal changes for random lines translating or rotating in depth compared with stationary control. Group data of three monkeys (M1, M3, and M5) are shown. D, Percentage of MR signal changes (relative to fixation) for action, static, and scrambled conditions. Group data of three monkeys (M1, M3, and M5) are shown. $\boldsymbol{E}$, Percentage of MR signal changes for goal-directed and mimicked actions and their respective static controls compared with the fixation baseline. Group data of two monkeys (M3 and M5) are shown. $\boldsymbol{F}$, Percentage of MR signal changes (relative to fixation) for hand actions and its static control and for moving and static objects. Group data of two monkeys (M3 and M5) are shown. Data in $\boldsymbol{A}-\boldsymbol{C}$ are derived from two hemispheres; data in $\boldsymbol{D}-\boldsymbol{F}$ are derived from the left hemisphere. Conventions are as in Figure 9. 


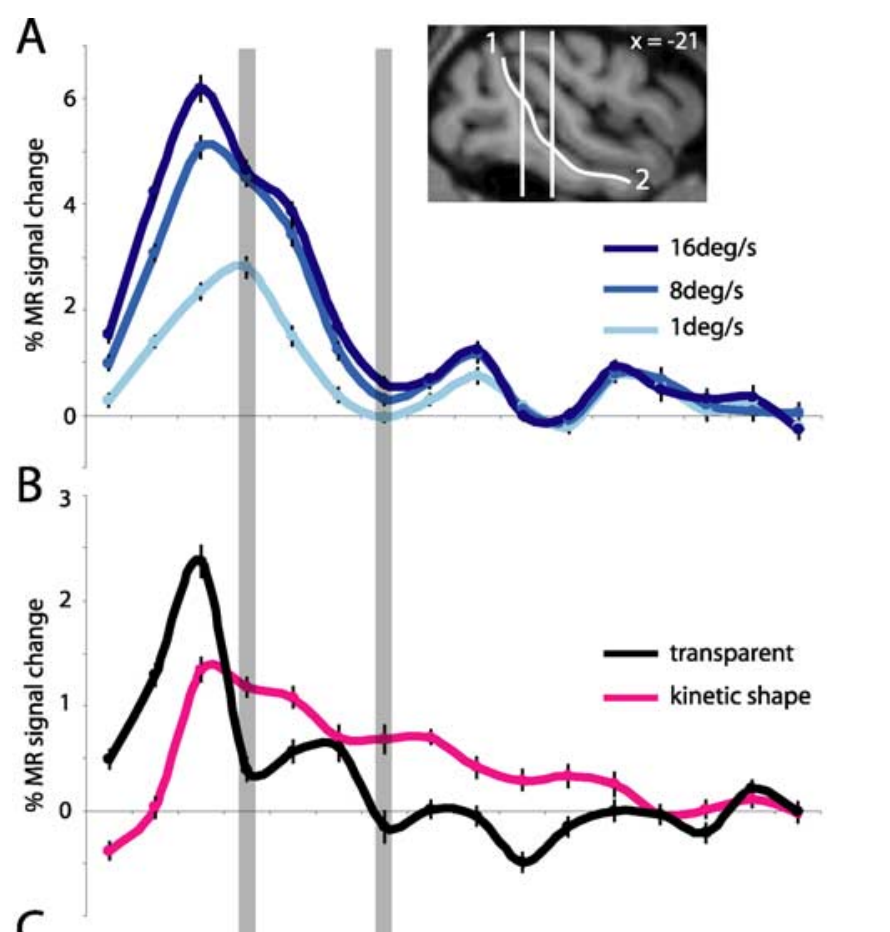

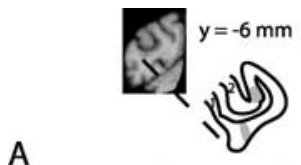
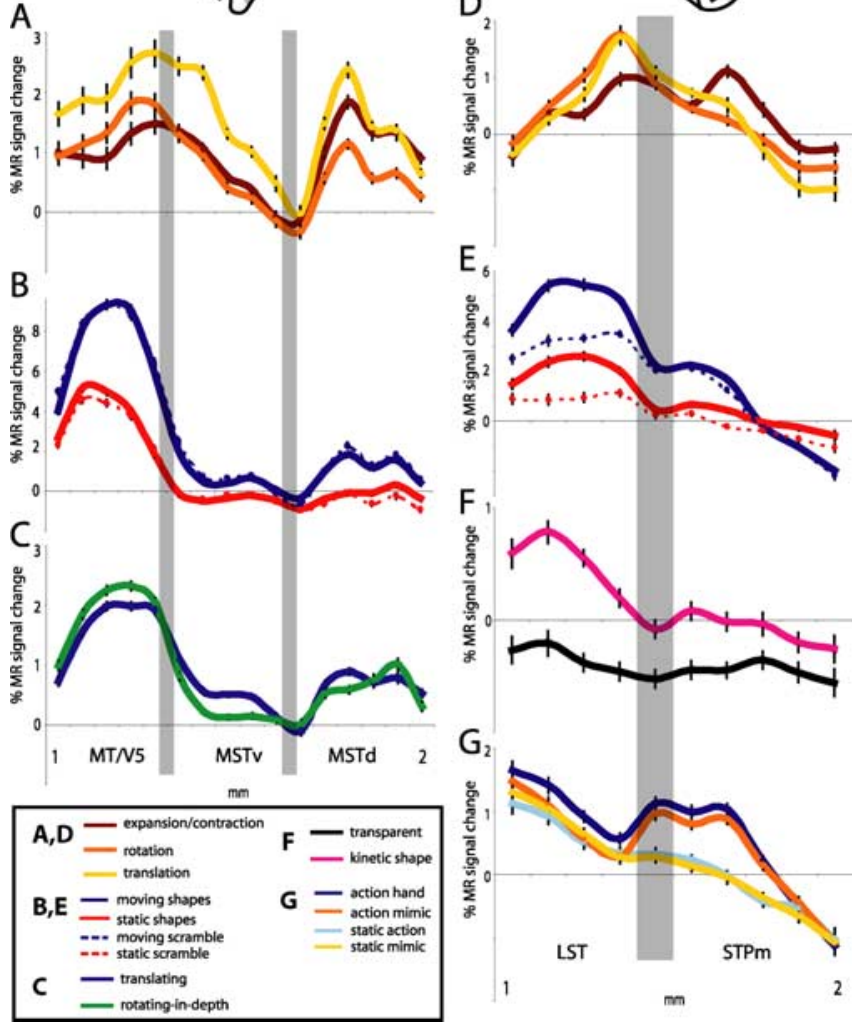

Figure 12. Line plots of MR activity in different conditions relative to baseline across the STS in coronal sections at level $-6(\boldsymbol{A}-\boldsymbol{C})$ and level $+3(\boldsymbol{D}-\boldsymbol{G})$. Group data (left hemisphere) from the optic flow test $(\boldsymbol{A}, \boldsymbol{D})$, moving-shapes test $(\boldsymbol{B}, \boldsymbol{E})$, structure-from-motion test $(\boldsymbol{C})$, shapefrom-motion test $(\boldsymbol{F})$, and goal-directed action test $(\boldsymbol{G})$ with conditions as indicated in the bottom left panel are shown. Baseline is the static plus condition $(\boldsymbol{A}, \boldsymbol{D})$, fixation $(\boldsymbol{B}, \boldsymbol{E}, \boldsymbol{G})$, static condition $(\boldsymbol{C})$, and uniform motion $(\boldsymbol{F})$. Gray vertical lines indicate boundaries (from Fig. 5). The insets show coronal sections plus a schematic plot of the STS with start and end points of the line plots and location of the borders (gray hatching).

motion-sensitive parts of the IT complex including area TEO, which was tentatively located lateral and posterior from the LST region (Fig. 5, black dashed outline).

Our results indicate that LST is a motion region. It responds more vigorously to a wide variety of the moving stimuli than to their static counterparts. This motion sensitivity of the LST region was consistently observed in all monkeys. In addition, it exhibits strong direction adaptation, suggesting that it may house direction-selective neurons. In fact, the LST region is a higherorder motion region with large RFs, like the MST and STPm regions. In that respect, the absence of retinotopic organization is not surprising. Most cortical regions with large RFs have little or no retinotopic organization (Bruce et al., 1981; Tanaka et al., 1991). It appears that the LST region has a large central representation, as described in the single-cell study of Hikosaka (1998), although we cannot exclude the possibility of a segregated representation of central and peripheral visual fields, like area FST.

The LST region is sensitive to kinetic shapes, relative to uniform motion and transparent controls. This might reflect an invariant processing of two-dimensional shape, whether static, moving, or kinetic, as observed in IT cortex (Sary et al., 1993), or in V4 (Mysore et al., 2005, 2006). Alternatively, it might represent sensitivity to opponent motion patterns, which as suggested rep- 
resents an important step between early motion processing and biological motion selectivity (Giese and Poggio, 2003). In this respect, it has some similarities with area FST, although area FST is more sensitive to two-dimensional and even more so to threedimensional shape from motion but is less sensitive to moving shapes (as opposed to moving scrambled images) than the LST region.

The LST region is also involved in the visual processing of actions: it responds more to actions than its static and scrambled controls. Also, it reacts more to a grasping than to a moving object (relative to static controls). This finding is in agreement with single-cell data of Perrett et al. (1985) demonstrating responses of single neurons in this region to grasping with hands. The LST region seems to act in concert with the STPm region with respect to action processing: although the LST region is shape sensitive, the STPm region is not. Thus, these two regions might use the two strategies put forward by Giese and Poggio (2003) for computing actions: changes in shape over time and changes in motion over space. The LST region, however, is the only element of the pair that seems to integrate action with object identity.

\section{A location for STPm and functional properties for FST}

The present study indicates that the STP region is most responsive to motion in its middle portion (Figs. 2, 6) and that this sensitivity includes both simple translation (Fig. 9A-C) and optic flow (Fig. $10 A$ ) components. Although several single-cell studies have documented STP responsiveness to various types of motion, including translation and optic flow components (Bruce et al., 1981; Perrett et al., 1985; Oram et al., 1993; Oram and Perrett, 1994; Anderson and Siegel, 1999), it has been difficult to locate the subregion in which these responses were most prevalent. Our MR findings suggest that simple motion responses predominate in the middle rather than in the rostral part of upper bank of the STS. The STP region has been traditionally associated (for review, see Puce and Perrett, 2003) with the processing of actions and biological motion. The present report, while confirming this view, shows that it shares this sensitivity with parts of the lower bank.

Our results also provide firm evidence for functional characteristics of area FST, which has hardly been explored with singlecell studies. Area FST exhibits clear direction adaptation suggesting that many of its neurons are direction selective. Yet Desimone and Ungerleider (1986) reported that only $30 \%$ of the FST cells were direction selective, but this might reflect the limitations of the stimuli used to assess direction selectivity. Alternatively, the direction adaptation might reflect the presence of opponent motion-selective neurons such as hypothesized by Giese and Poggio (2003).

Area FST is not only involved in the processing of action, but it also has rather specific properties related to the processing of two- and three-dimensional shape from motion (Sereno et al., 2002, 2005; Vanduffel et al., 2002). In fact, except for MT/V5 itself, area FST is the only motion-sensitive STS region that processes three-dimensional shape from motion, specified by random lines.

\section{Experimental strategy for charting cortical regions using fMRI}

Our strategy for defining (Fig. 5) and functionally characterizing six motion-sensitive regions in monkey STS using fMRI involved five steps: (1) localization of the motion-sensitive cortex within the STS; (2) definition of specific regions by a few prototypical contrasts; (3) confirmation of the motion sensitivity by the direction adaptation; (4) validation of the parcellation by independent statistical testing using new contrasts not used in steps 1-3; and (5) demonstration of sharp changes at the boundaries.

Further validation is provided by the match between the overall organization of MT/V5 and its satellites described here and in previous studies. The central representation of MT/V5 borders on areas MSTv and FST in the fundus of the STS, whereas area MSTd is located in the upper bank of the caudal STS (Desimone and Ungerleider, 1986). It is likely that the part of the STS flattened in the present study may lack the most posterior part of the MST area. The unresponsive cortex in the present study (supplemental Fig. S1, available at www.jneurosci.org as supplemental material) may correspond to the posterior, wider part of the mostly unresponsive region of Hikosaka et al. (1988). Our data agree with the subdivision of area MST put forward by Tanaka et al. (1993) and more generally with all schemes subdividing area MST into two parts (Komatsu and Wurtz, 1988; Boussaoud et al., 1990; Felleman and Van Essen, 1991). It should be noted, however, that increasing the battery of stimuli and the spatial resolution of fMRI techniques may permit further functional distinctions, thereby increasing the number of subdivisions of area MST (Lewis and Van Essen, 2000).

Finally, additional validation of our parcellation is provided by the agreement between the functional properties revealed by the present fMRI tests and by previous single-cell studies as mentioned in Results.

\section{Functional organization of the motion pathway in monkey STS}

Area MT/V5 represents the first step in motion processing in monkey STS. Subsequent, higher-order regions are characterized by a crude retinotopy and larger RFs, in agreement with singlecell studies (Bruce et al., 1981; Desimone and Ungerleider, 1986). Furthermore, beyond MT/V5, motion-sensitive regions respond less to transparent motion compared with uniform motion. The decrease in response to transparent compared with uniform motion has been considered a characteristic of higher-order motion regions (Qian and Andersen, 1994; Sunaert et al., 1999; Braddick et al., 2000). A number of the motion-sensitive regions, notably MT/V5, FST, and LST, respond to static stimuli, yet only LST is sensitive to two-dimensional shape, because it responds more to intact than scrambled images of objects.

Our results provide a clear indication that multiple motion pathways arise from MT/V5. The MST areas respond very differently from the group that includes FST, LST, and STPm to action videos, kinetic shapes, or three-dimensional structure from motion compared with two-dimensional motion displays (Figs. 9, 10). Although the MST areas display spatial summation, FST, LST, and STPm show little or no spatial summation. This suggest that, in addition to the MST areas involved in control of locomotion and pursuit/tracking of small objects (Saito et al., 1986; Komatsu and Wurtz, 1988), MT/V5 projects into a more ventral and rostral pathway dedicated to the visual processing of action (supplemental Fig. S5, available at www.jneurosci.org as supplemental material): all four areas (MT/V5, FST, LST, and STPm) react more strongly to actions than to static and dynamic control stimuli. Within this pathway, responses to lower-level dynamic stimuli such as presented in the scrambled condition decrease, whereas the responses to opponent motion stimuli begin to appear. Furthermore, the different regions of the "action" pathway have distinct characteristics: area FST is sensitive to three-dimensional shape from motion [which bears some relationship with biological motion processing (Vanrie and Verfaillie, 2006)], the LST region is sensitive 
to the combination of action and object identity, and STPm is sensitive to action as such.

\section{References}

Albright TD (1984) Direction and orientation selectivity of neurons in visual area MT of the macaque. J Neurophysiol 52:1106-1130.

Anderson KC, Siegel RM (1999) Optic flow selectivity in the anterior superior temporal polysensory area, STPa, of the behaving monkey. J Neurosci 19:2681-2692.

Boussaoud D, Ungerleider LG, Desimone R (1990) Pathways for motion analysis: cortical connections of the medial superior temporal and fundus of the superior temporal visual areas in the macaque. J Comp Neurol 296:462-495.

Braddick OJ, O’Brien JM, Wattam-Bell J, Atkinson J, Turner R (2000) Form and motion coherence activate independent, but not dorsal/ventral segregated, networks in the human brain. Curr Biol 10:731-734.

Bruce CJ, Desimone R, Gross CG (1981) Visual properties of neurons in a polysensory area in superior temporal sulcus of the macaque. J Neurophysiol 46:369-384.

Buckner RL, Koutstaal W, Schacter DL, Dale AM, Rotte MR, Rosen BR (1998) Functional-anatomic study of episodic retrieval. II. Selective averaging of event-related fMRI trials to test the retrieval success hypothesis. NeuroImage 7:163-175.

Chef d'Hotel C, Hermosillo G, Faugeras O (2002) Flows of diffeomorphisms for multimodal image registration. Proc IEEE S Bio Im 7- 8:21-28.

Cheng K, Hasegawa T, Saleem KS, Tanaka K (1994) Comparison of neuronal selectivity for stimulus speed, length, and contrast in the prestriate visual cortical areas V4 and MT of the macaque monkey. J Neurophysiol 71:2269-2280.

Cusick CG (1997) The superior temporal polysensory region in monkeys. In: Cerebral cortex 12. Extrastriate cortex in primates (Rockland K, Kaas JH, Peters A, eds), pp 435-468. New York: Plenum.

Denys K, Vanduffel W, Fize D, Nelissen K, Peuskens H, Van Essen D, Orban GA (2004) The processing of visual shape in the cerebral cortex of human and nonhuman primates: a functional magnetic resonance imaging study. J Neurosci 24:2551-2565.

Desimone R, Ungerleider LG (1986) Multiple visual area in the caudal superior temporal sulcus of the macaque. J Comp Neurol 248:164-189.

Distler C, Boussaoud D, Desimone R, Ungerleider LG (1993) Cortical connections of inferior temporal area TEO in macaque monkeys. J Comp Neurol 334:125-150.

Dubner R, Zeki SM (1971) Response properties and receptive fields of cells in an anatomically defined region of the superior temporal sulcus in the monkey. Brain Res 35:528-532.

Duffy CJ, Wurtz RH (1991) Sensitivity of MST neurons to optic flow stimuli. I. A continuum of response selectivity to large-field stimuli. J Neurophysiol 65:1329-1345.

Dukelow SP, DeSouza JF, Culham JC, van den Berg AV, Menon RS, Vilis T (2001) Distinguishing subregions of the human MT+ complex using visual fields and pursuit eye movements. J Neurophysiol 86:1991-2000.

Eifuku S, Wurtz RH (1999) Response to motion in extrastriate area MSTl: disparity sensitivity. J Neurophysiol 82:2462-2475.

Felleman DJ, Van Essen DC (1991) Distributed hierarchical processing in the primate cerebral cortex. Cereb Cortex 1:1-47.

Fize D, Vanduffel W, Nelissen K, Denys K, Chef d'Hotel C, Faugeras O, Orban GA (2003) The retinotopic organization of primate dorsal V4 and surrounding areas: an fMRI study in awake monkeys. J Neurosci 23:7395-7406.

Giese MA, Poggio T (2003) Neural mechanisms for the recognition of biological movements. Nat Rev Neurosci 4:179-192.

Grafton ST, Arbib MA, Fadiga L, Rizzolatti G (1996) Localization of grasp representations in humans by positron emission tomography. 2. Observation compared with imagination. Exp Brain Res 112:103-111.

Graziano MSA, Andersen RA, Snowden RJ (1994) Tuning of MST neurons to spiral motions. J Neurosci 14:54-67.

Handwerker DA, Ollinger JM, D'Esposito M (2004) Variation in BOLD hemodynamic responses across subjects and brain regions and their effects on statistical analyses. NeuroImage 21:1639-1651.

Harel N, Zhao F, Wang P, Kim SG (2002) Cortical layer specificity of BOLD and CBV fMRI signals at ultra-high resolution. Paper presented at 10th Annual Meeting of the International Society for Magnetic Resonance in Medicine, Honolulu, HI, May.

Hikosaka K (1998) Representation of foveal visual fields in the ventral bank of the superior temporal sulcus in the posterior inferotemporal cortex of the macaque monkey. Behav Brain Res 96:101-113.

Hikosaka K, Iwai E, Saito H, Tanaka K (1988) Polysensory properties of neurons in the anterior bank of the caudal superior temporal sulcus of the macaque monkey. J Neurophysiol 60:1615-1637.

Huk AC, Ress D, Heeger DJ (2001) Neuronal basis of the motion aftereffect reconsidered. Neuron 32:161-172.

Huk AC, Dougherty RF, Heeger DJ (2002) Retinotopy and functional subdivision of human areas MT and MST. J Neurosci 22:7195-7205.

Janssen P, Vogels R, Orban GA (2000) Selectivity for 3D shape that reveals distinct areas within macaque inferior temporal cortex. Science 288:2054-2056.

Komatsu H, Wurtz RH (1988) Relation of cortical areas MT and MST to pursuit eye movements. I. Localization and visual properties of neurons. J Neurophysiol 60:580-603.

Kourtzi Z, Kanwisher N (2000) Cortical regions involved in perceiving object shape. J Neurosci 20:3310-3318.

Lagae L, Raiguel S, Orban GA (1993) Speed and direction selectivity of macaque middle temporal neurons. J Neurophysiol 69:19-39.

Lagae L, Maes H, Raiguel S, Xiao DK, Orban GA (1994) Responses of macaque STS neurons to optic flow components: a comparison of areas MT and MST. J Neurophysiol 71:1597-1626.

Leite FP, Tsao D, Vanduffel W, Fize D, Sasaki Y, Wald LL, Dale AM, Kwong KK, Orban GA, Rosen BR, Tootell RB, Mandeville JB (2002) Repeated fMRI using iron oxide contrast agent in awake, behaving macaques at 3 tesla. NeuroImage 16:283-294.

Lewis JW, Van Essen DC (2000) Mapping of architectonic subdivisions in the macaque monkey, with emphasis on parieto-occipital cortex. J Comp Neurol 428:79-111.

Logothetis NK, Guggenberger H, Peled S, Pauls J (1999) Functional imaging of the monkey brain. Nat Neurosci 2:555-562.

Mandeville JB, Marota JJ (1999) Vascular filters of functional MRI: spatial localization using BOLD and CBV contrast. Magn Reson Med 42:591-598.

Maunsell JHR, Van Essen DC (1983) Functional properties of neurons in middle temporal visual area of the macaque monkey. I. Selectivity of stimulus direction, speed, and orientation. J Neurophysiol 49:1127-1147.

Maunsell JHR, Van Essen DC (1987) Topographic organization of the middle temporal visual area in the macaque monkey: representational biases and the relationship to callosal connections and myeloarchitectonic boundaries. J Comp Neurol 266:535-555.

Miezin FM, Maccotta L, Ollinger JM, Petersen SE, Buckner RL (2000) Characterizing the hemodynamic response: effects of presentation rate, sampling procedure, and the possibility of ordering brain activity based on relative timing. NeuroImage 11:735-759.

Mikami A, Newsome WT, Wurtz RH (1986) Motion selectivity in macaque visual cortex. I. Mechanisms of direction and speed selectivity in extrastriate area MT. J Neurophysiol 55:1308-1327.

Mysore SG, Raiguel SE, Vogels R, Orban GA (2005) Cue invariance in V4 for static and moving shapes. Soc Neurosci Abstr 31:46.18.

Mysore SG, Vogels R, Raiguel SE, Orban GA (2006) Processing of kinetic boundaries in macaque V4. J Neurophysiol 95:1864-1880.

Nelissen K, Luppino G, Vanduffel W, Rizzolatti G, Orban GA (2005) Observing others: multiple action representation in the frontal lobe. Science 310:332-336.

Oram MW, Perrett DI (1994) Responses of anterior superior temporal polysensory (STPa) neurons to "biological motion" stimuli. J Cogn Neurosci 6:99-116.

Oram MW, Perrett DI, Hietanen JK (1993) Directional tuning of motionsensitive cells in the anterior superior temporal polysensory area of the macaque. Exp Brain Res 97:274-294.

Orban GA, Lagae L, Raiguel S, Xiao DK, Maes H (1995) The speed tuning of medial superior temporal (MST) cell responses to optic-flow components. Perception 24:269-285.

Perrett DI, Smith PA, Mistlin AJ, Chitty AJ, Head AS, Potter DD, Broennimann R, Milner AD, Jeeves MA (1985) Visual analysis of body movements by neurones in the temporal cortex of the macaque monkey: a preliminary report. Behav Brain Res 16:153-170.

Perrett DI, Harries MH, Bevan H, Thomas S, Benson PJ, Mistlin AJ, Chitty AJ, Hietanen JK, Ortega JE (1989) Frameworks of analysis for the neural representation of animate objects and actions. J Exp Biol 146:87-113.

Puce A, Perrett DI (2003) Electrophysiology and brain imaging of biological motion. Philos Trans R Soc Lond B Biol Sci 358:435-445. 
Qian N, Andersen RA (1994) Transparent motion perception as detection of unbalanced motion signals. II. Physiology. J Neurosci 14:7367-7380.

Raiguel S, Van Hulle M, Xiao DK, Marcar VL, Orban GA (1995) Shape and spatial distribution of receptive fields and antagonistic motion surrounds in the middle temporal area (V5) of the macaque. Eur J Neurosci 7:2064-2082.

Raiguel S, Van Hulle MM, Xiao DK, Marcar VL, Lagae L, Orban GA (1997) Size and shape of receptive fields in the medial superior temporal area (MST) of the macaque. NeuroReport 8:2803-2808.

Regan D (1989) Orientation discrimination for objects defined by relative motion and objects defined by luminance contrast. Vision Res 10:1389-1400.

Robson MD, Dorosz JL, Gore JC (1998) Measurements of the temporal fMRI response of the human auditory cortex to trains of tones. NeuroImage 7:185-198.

Saito H, Yukie M, Tanaka K, Hikosaka K, Fukada Y, Iwai E (1986) Integration of direction signals of image motion in the superior temporal sulcus of the macaque monkey. J Neurosci 6:145-157.

Saleem KS, Suzuki W, Tanaka K, Hashikawa T (2000) Connections between anterior inferotemporal cortex and superior temporal sulcus regions in the macaque monkey. J Neurosci 20:5083-5101.

Sary G, Vogels R, Orban GA (1993) Cue-invariant shape selectivity of macaque inferior temporal neurons. Science 260:995-997.

Sawamura H, Georgieva S, Vogels R, Vanduffel W, Orban G (2005) Using functional magnetic resonance imaging to assess adaptation and size invariance of shape processing by humans and monkeys. J Neurosci 25:4294-4306.

Schacter DL, Buckner RL, Koutstaal W, Dale AM, Rosen BR (1997) Late onset of anterior prefrontal activity during true and false recognition: an event-related fMRI study. NeuroImage 6:259-269.

Seltzer B, Pandya DN (1978) Afferent cortical connections and architectonics of the superior temporal sulcus and surrounding cortex in the rhesus monkey. Brain Res 149:1-24.

Sereno ME, Trinath T, Augath M, Logothetis NK (2002) Three-dimensional shape representation in monkey cortex. Neuron 33:635-652.

Sereno ME, Augath M, Logothetis NK (2005) Differences in processing of $3 \mathrm{D}$-shape from multiple cues in monkey cortex revealed by $\mathrm{fMRI}$. Soc Neurosci Abstr 31:362.9.

Sunaert S, Van Hecke P, Marchal G, Orban GA (1999) Motion-responsive regions of the human brain. Exp Brain Res 127:355-370.

Tanaka K, Hikosaka K, Saito H, Yukie M, Fukada Y, Iwai E (1986) Analysis of local and wide-field movements in the superior temporal visual areas of the macaque monkey. J Neurosci 6:134-144.

Tanaka K, Fukada Y, Saito H (1989) The underlying mechanisms of the response specificity of expansion/contraction and rotation cells in the dorsal part of the medial superior temporal area of the macaque monkey. J Neurophysiol 62:642-656.

Tanaka K, Saito H, Fukada H, Moriya M (1991) Coding visual images of objects in the inferotemporal cortex of the macaque monkey. J Neurophysiol 66:170-189.

Tanaka K, Sugita Y, Moriya M, Saito H (1993) Analysis of object motion in the ventral part of the medial superior temporal area of the macaque visual cortex. J Neurophysiol 69:128-142.

Todd JT (1984) The perception of three-dimensional structure from rigid and nonrigid motion. Percept Psychophys 36:97-103.

Tolias AS, Smirnakis SM, Augath MA, Trinath T, Logothetis NK (2001) Motion processing in the macaque: revisited with functional magnetic resonance imaging. J Neurosci 21:8594-8601.

Ungerleider LG, Desimone R (1986a) Projections to the superior temporal sulcus from the central and peripheral field representations of V1 and V2. J Comp Neurol 248:147-163.

Ungerleider LG, Desimone R (1986b) Cortical connections of visual area MT in the macaque. J Comp Neurol 248:190-222.

Unno S, Nagasaka Y, Inoue M, Mikami A (2002) Relative contributions of the upper and lower banks of macaque superior temporal sulcus to perception of shapes defined by motion signal. Soc Neurosci Abstr 28:160.9.

Van Doorn AJ, Koenderink JJ (1982) Spatial properties of the visual detectability of moving spatial white noise. Exp Brain Res 45:189-195.

Vanduffel W, Fize D, Mandeville JB, Nelissen K, Van Hecke P, Rosen BR, Tootell RB, Orban GA (2001) Visual motion processing investigated using contrast agent-enhanced fMRI in awake behaving monkeys. Neuron 32:565-577.

Vanduffel W, Fize D, Peuskens H, Denys K, Sunaert S, Todd JT, Orban GA (2002) Extracting 3D from motion: differences in human and monkey intraparietal cortex. Science 298:413-415.

Van Essen DC (2004) Organization of visual areas in macaque and human cerebral cortex. In: The visual neurosciences (Chalupa L, Werner JS, eds), pp 507-521. Cambridge, MA: MIT.

Van Essen DC (2005) A population-average, landmark- and surface-based (PALS) atlas of human cerebral cortex. NeuroImage 28:635-662.

Van Essen DC, Maunsell JH, Bixby JL (1981) The middle temporal visual area in the macaque: myeloarchitecture, connections, functional properties and topographic organization. J Comp Neurol 199:293-326.

Van Oostende S, Sunaert S, Van Hecke P, Marchal G, Orban GA (1997) The kinetic occipital (KO) region in man: an fMRI study. Cereb Cortex 7:690-701.

Vanrie J, Verfaillie K (2006) Perceiving depth in point-light actions. Percept Psychophys, in press.

Zeki SM (1974) Functional organization of a visual area in the posterior bank of the superior temporal sulcus of the rhesus monkey. J Physiol (Lond) 236:549-573.

Zhao F, Wang P, Hendrich K, Kim SG (2005) Spatial specificity of cerebral blood volume-weighted fMRI responses at columnar resolution. NeuroImage 27:416-424.

Zhao F, Wang P, Hendrich K, Ugurbil K, Kim SG (2006) Cortical layerdependent BOLD and CBV responses measured by spin-echo and gradient-echo fMRI: insights into hemodynamic regulation. NeuroImage 30:1149-1160. 